

\title{
Plates with incompatible prestrain of high order
}

\author{
Marta Lewicka, Annie Raoult, Diego Ricciotti
}

\section{To cite this version:}

Marta Lewicka, Annie Raoult, Diego Ricciotti. Plates with incompatible prestrain of high order. Annales de l'Institut Henri Poincaré, 2017, 34, pp.1883-1912. 10.1016/j.anihpc.2017.01.003 hal01138338

\section{HAL Id: hal-01138338 \\ https://hal.science/hal-01138338}

Submitted on 1 Apr 2015

HAL is a multi-disciplinary open access archive for the deposit and dissemination of scientific research documents, whether they are published or not. The documents may come from teaching and research institutions in France or abroad, or from public or private research centers.
L'archive ouverte pluridisciplinaire HAL, est destinée au dépôt et à la diffusion de documents scientifiques de niveau recherche, publiés ou non, émanant des établissements d'enseignement et de recherche français ou étrangers, des laboratoires publics ou privés. 


\title{
PLATES WITH INCOMPATIBLE PRESTRAIN OF HIGHER ORDER
}

\author{
MARTA LEWICKA, ANNIE RAOULT, AND DIEGO RICCIOTTI
}

\begin{abstract}
We study the effective elastic behaviour of the incompatibly prestrained thin plates, characterized by a Riemann metric $G$ on the reference configuration. We assume that the prestrain is "weak", i.e. it induces scaling of the incompatible elastic energy $E^{h}$ of order less than $h^{2}$ in terms of the plate's thickness $h$.

We essentially prove two results. First, we establish the $\Gamma$-limit of the scaled energies $h^{-4} E^{h}$ and show that it consists of a von Kármán-like energy, given in terms of the first order infinitesimal isometries and of the admissible strains on the surface isometrically immersing $G_{2 \times 2}$ (i.e. the prestrain metric on the midplate) in $\mathbb{R}^{3}$. Second, we prove that in the scaling regime $E^{h} \sim h^{\beta}$ with $\beta>2$, there is no other limiting theory: if inf $h^{-2} E^{h} \rightarrow 0$ then $\inf E^{h} \leq C h^{4}$, and if $\inf h^{-4} E^{h} \rightarrow 0$ then $G$ is realizable and hence $\min E^{h}=0$ for every $h$.
\end{abstract}

\section{Contents}

1. Introduction 1

1.1. Some background in dimension reduction for thin structures 2

1.2. The set-up and notation 4

1.3. Some previous directly related results 5

1.4. New results of this work 6

1.5. Acknowledgments 7

2. The scaling and approximation lemmas $\quad 7$

3. The lower bound 10

4. The upper bound 17

5. Discussion of the von Kármán-like functional (3.5) 23

6. The scaling optimality 25

7. Two examples 27

8. Appendix: a proof of Corollary 2.3 30

References $\quad 32$

\section{INTRODUCTION}

The purpose of this paper is to study effective elastic behaviour of the incompatibly prestressed thin plates $\Omega^{h}$, characterized by a Riemann metric $G$ given on their reference configuration. The incompatibility is measured through the energy $E^{h}$ given below (sometimes called the "non-Euclidean" elastic energy).

Date: 23 March, 2015. 
We will be concerned with the regime of curvatures of $G$ which yields the incompatibility rate of order higher than $h^{2}$, in plate's thickness $h$. Indeed, in paper [6] we analyzed the scaling $\inf E^{h} \sim h^{2}$ and proved that it only occurs when the metric $G_{2 \times 2}$ on the mid-plate can be isometrically immersed in $\mathbb{R}^{3}$ with the regularity $W^{2,2}$ and when, at the same time, the three appropriate Riemann curvatures of $G$ do not vanish identically (for details, see below). The relevant residual theory, obtained through $\Gamma$-convergence, yielded a bending Kirchhoff-like residual energy.

In the present paper we assume that:

$$
h^{-2} \text { inf } E^{h} \rightarrow 0
$$

and prove that the only nontrivial residual theory in this regime is a von Kármán-like energy, valid when $\inf E^{h} \sim h^{4}$. It further turns out that this scaling is automatically implied by (1.1) and inf $E^{h} \neq 0$. Indeed, we show that if (1.1) then $h^{-4} \inf E^{h} \leq C$, and that $h^{-4}$ inf $E^{h} \rightarrow 0$ if and only if $G$ is immersible whereas trivially $\min E^{h}=0$ for all $h$.

This scale separation is contrary to the findings of [29] valid in the Euclidean case of $G=\mathrm{Id}_{3}$, where the residual energies are driven by presence of applied forces $f^{h} \sim h^{\alpha}$. In that context, three distinct limiting theories have been obtained for $E^{h} \sim h^{\beta}$ with $\beta>2$ (equivalently $\alpha>2$ ). Namely: $\beta \in(2,4)$ corresponded to the linearized Kirchhoff (nonlinear bending) model subject to a nonlinear constraint on the displacements, $\beta=4$ to the classical von Kármán model, and $\beta>4$ to the linear elasticity. The present results are also contrary to the higher order hierarchy of scalings and of the resulting elastic theories of shells, as derived by an asymptotic calculus in [45]. The difference is due to the fact that while the magnitude of external forces is adjustable at will, it seems not to be the case for the interior mechanism of a given metric $G$ which does not depend on $h$. In fact, it is the curvature tensor of $G$ that induces the nontrivial stresses in the thin film. The Riemann tensor of a three-dimensional metric has only six independent components, namely the six sectional curvatures created out of the three principal directions, which further fall into two categories: including or excluding the thin direction variable. The simultaneous vanishing of curvatures in each of such categories correspond to the two scenarios at hand in terms of the scaling of the residual energy.

1.1. Some background in dimension reduction for thin structures. Early attempts for replacing the three-dimensional model of a thin elastic structure with planar mid-surface at rest, by a two-dimensional model, were based on a priori simplifying assumptions on the deformations and on the stresses. Later, the natural idea of using the thickness as a small parameter and of establishing a limit model was largely explored; we refer in particular to the works by Ciarlet and Destuynder who set the method in the appropriate framework of the weak formulation of the boundary value problems $[12,13]$, proved convergence to the linear plate model [23] in the context of linearized elasticity, and obtained formally the von Kármán plate model from finite elasticity [9]. See also $[55,56]$ for the time-dependent case and convergence results, and [10] for a comprehensive list of references.

The issue of deriving two-dimensional models valid for large deformations, by means of an asymptotic formalism, was subsequently tackled by Fox, Simo and the second author in [26]. They showed, in the context of the Saint Venant-Kirchhoff materials subject to appropriate 
boundary conditions, how to recover a hierarchy of four models. This hierarchy, driven by the order of magnitude of the applied loads, consisted of: the nonlinear membrane model, the inextensional bending model, the von Kármán model and the linear plate model. The models thus obtained still required a justification through rigorous convergence results. In [34], Le Dret and the second author used the variational point of view and proved $\Gamma$-convergence of the three-dimensional elastic energies to a nonlinear membrane energy, valid for loads of magnitude of order 1 . We remark that the expression of the limiting stored energy therein consisted of quasiconvexification of the three-dimensional energy, first minimized with respect to the normal stretches. This allowed to recover the degeneracy under compression; a feature that is otherwise missed by formal expansions. We further mention that for $3 \mathrm{~d} \rightarrow 1 \mathrm{~d}$ reduction a similar point of view had been introduced by Acerbi, Buttazzo and Percivale in [1].

A key-point for deriving rigorously the above mentioned nonlinear bending model has been the geometric rigidity result due to Friesecke, James and Müller [28]. In the similar spirit, the same authors justified the von Kármán model, the linear model [29] and also they introduced novel intermediate models, in particular in the range of energies - or equivalently of loadings - between the scaling responsible for bending $(\beta=2)$ and the von Kármán scaling $(\beta=4)$. In this range of models, the three-dimensional stored energy appears in the limit stored energy through its second derivative at rest. Scaling the energy with exponents $\beta$ other than integers had been explored for the membrane to bending range in [19] leading to convergence results for $0<\beta<5 / 3$ while the regime $5 / 3 \leq \beta<2$ remains open and is conjectured to be relevant for crumpling of elastic sheets. Other significant extensions concern derivation of limit theories for incompressible materials [17, 18, 63, 47], for heterogeneous materials [61], through establishing convergence of equilibria rather than strict minimizers $[52,62,36,53,37]$, and finally for shallow shells [39].

Extension of the above variational method valid in the framework of the large deformation model was conducted in parallel for slender structures whose midsurface at rest is non-planar. The first result by the second author and Le Dret [35] relates to scaling $\beta=0$ and models membrane shells: the limit stored energy depends then only on the stretching and shearing produced by the deformation on the midsurface. Another study is due to Friesecke, James, Mora and Müller [27] who analyzed the case $\beta=2$. This scaling corresponds to a flexural shell model, where the only admissible deformations are those preserving the midsurface metric. The limit energy depends then on the change of curvature produced by the deformation. Further, the first author, Mora and Pakzad derived the relevant linear theories $(\beta>4)$ and the von Kármán-like theories $(\beta=4)$ in [41], and subsequently proceeded to finalize the analysis for elliptic shells in the full regime $\beta>2$ in [42]. A similar analysis has been performed in case of the developable shells in [32] leading to the proof of the collapse of all residual theories to the linear theory when $\beta>2$. Following these findings, a conjecture was made in [45] about the infinite hierarchy of shell models and the various possible limiting scenarios differentiatied by rigidity properties of shells. Let us recall that a comprehensive body of work had been previously devoted to the asymptotic derivation of shell models in the small displacement regime under clear hypotheses on the model taken for granted, threedimensional or already two-dimensional and containing the thickness as a parameter. Several models were recovered by Ciarlet and coauthors $[14,15,16]$, by Destuynder $[22,24]$ and by Sanchez-Palencia and coauthors $[59,60,8,7,50]$. Sanchez-Palencia, in particular, theorized 
the role and interplay of the midsurface geometry and of the boundary conditions [58], as well as underlined the singular perturbation behavior. We refer to [11] for many additional references.

Most recently, there has been a sustained interest in studying similar problems where the shape formation is not driven by exterior forces but rather by the internal prestrain caused by e.g. growth, swelling, shrinkage or plasticity [33, 25, 62]. Variants of a thin plate theory can be used to study the self-similar structures which form due to variations in an intrinsic metric of a surface that is asymptotically flat at infinity [2], and also in the case of a circular disk with edge-localized growth [25], or in the shape of a long leaf [48]. Ben Amar and coauthors formally derived a variant of the Föppl-von Kármán equilibrium equations from finite incompressible elasticity [20,21]: they use the multiplicative decomposition of the gradient proposed in [57] similar to ours. They take cockling of paper, grass blades and sympatelous flowers as examples [21,5].

A systematic study of the possible limit problems when a target metric is prescribed was undertaken by the first author and collaborators: a residually strained version of the Kirchhoff theory for plates was, for the first time, rigorously derived in [44] under the assumption that the target metric is independent of thickness. This analysis was completed in [6], resulting in a necessary and sufficient condition that the elastic prestrained energy scales as $h^{2}$. The object of the present paper is to study higher order prestrains.

Let us also mention that in $[39,40,43]$ similar derivations were carried out under a different assumption on the asymptotic behavior of the prescribed metric, which also implied energy scaling $h^{\beta}$ in different regimes of $\beta>2$. In [39] it was shown that the resulting equations are identical to those postulated to account for the effects of growth in elastic plates [48] and used to describe the shape of a long leaf. In [43] a model with a Monge-Ampère constraint was derived and analysed from various aspects. Other results concerning the energy scaling for the materials with residual strain are derived in [4], where by imposing suitable boundary data, conditions of $[44,6]$ are not satisfied and hence the residual energy scales larger than $h^{2}$, depending on the type of these boundary data (see also [62]).

1.2. The set-up and notation. Let $\Omega$ be an open, bounded, smooth and simply connected subset of $\mathbb{R}^{2}$. For $0<h \ll 1$ we consider thin films $\Omega^{h}$ around the mid-plate $\Omega$ :

$$
\Omega^{h}=\left\{x=\left(x^{\prime}, x_{3}\right) ; x^{\prime} \in \Omega, x_{3} \in(-h / 2, h / 2)\right\} .
$$

Let $G: \bar{\Omega}^{h} \rightarrow \mathbb{R}^{3 \times 3}$ be a given smooth Riemann metric on $\Omega^{h}$, uniform through the thickness:

$$
G\left(x^{\prime}, x_{3}\right)=G\left(x^{\prime}\right) \text { for every }\left(x^{\prime}, x_{3}\right) \in \Omega^{h},
$$

and let $A=\sqrt{G}$ denote the unique positive definite symmetric square root of $G$. Consider the energy functional $E^{h}: W^{1,2}\left(\Omega^{h}, \mathbb{R}^{3}\right) \rightarrow \overline{\mathbb{R}}_{+}$defined as:

$$
E^{h}\left(u^{h}\right)=\frac{1}{h} \int_{\Omega^{h}} W\left(\nabla u^{h} A^{-1}\right) \mathrm{d} x .
$$

The nonlinear elastic energy density $W: \mathbb{R}^{3 \times 3} \rightarrow \overline{\mathbb{R}}_{+}$is a Borel measurable function, assumed to be $\mathcal{C}^{2}$ in a neighborhood of $S O(3)$ and to satisfy, for every $F \in \mathbb{R}^{3 \times 3}$, every $R \in S O(3)$ 
and with a uniform constant $c>0$, the conditions:

$$
W(R)=0, \quad W(R F)=W(F), \quad W(F) \geq c \operatorname{dist}^{2}(F, S O(3)) .
$$

The first condition states that the energy of a rigid motion is 0 , while the second is the frame invariance. They imply that $D W\left(\operatorname{Id}_{3}\right)=0$ and that $D^{2} W\left(\operatorname{Id}_{3}\right)(S, \cdot)=0$ for all skew symmetric matrices $S \in s o(3)$. The third assumption above reflects the quadratic growth of the density $W$ away from the energy well $S O(3)$. Note that these assumptions are not contradictory with the physical condition $W(F)=\infty$ for $\operatorname{det} F \leq 0$.

Throughout the paper, we use the following notation. Given a matrix $F \in \mathbb{R}^{3 \times 3}$, we denote its transpose by $F^{t}$, its symmetric part by $\operatorname{sym} F=\frac{1}{2}\left(F+F^{t}\right)$, and its skew part by skew $F=F-\operatorname{sym} F$. By $S O(n)=\left\{R \in \mathbb{R}^{n \times n} ; R^{t}=R^{-1}\right.$ and $\left.\operatorname{det} R=1\right\}$ we denote the group of special rotations, while $\operatorname{so}(n)=\left\{F \in \mathbb{R}^{n \times n}\right.$; $\left.\operatorname{sym} F=0\right\}$ is the space of skewsymmetric matrices. We use the matrix norm $|F|=\left(\operatorname{trace}\left(F^{t} F\right)\right)^{1 / 2}$, which is induced by the inner product $\left\langle F_{1}: F_{2}\right\rangle=\operatorname{trace}\left(F_{1}^{t} F_{2}\right)$. The $2 \times 2$ principal minor of a matrix $F \in \mathbb{R}^{3 \times 3}$ is denoted by $F_{2 \times 2}$. Conversely, for a given $F_{2 \times 2} \in \mathbb{R}^{2 \times 2}$, the $3 \times 3$ matrix with principal minor equal $F_{2 \times 2}$ and all other entries equal to 0 , is denoted by $\left(F_{2 \times 2}\right)^{*}$. All limits are taken as the thickness parameter $h$ vanishes, i.e. when $h \rightarrow 0$. Finally, by $C$ we denote any universal constant, independent of $h$.

1.3. Some previous directly related results. It has been proved in [44] that:

$$
\inf _{u^{h} \in W^{1,2}\left(\Omega^{h}, \mathbb{R}^{3}\right)} E^{h}\left(u^{h}\right)=0
$$

if and only if the Riemann curvature tensor of $G$ vanishes identically in $\Omega^{h}$, i.e.: $\operatorname{Riem}(G) \equiv 0$, and when (equivalently) the infimum above is achieved through a smooth isometric immersion $u^{h}$ of the metric $G$ on $\Omega^{h}$. Further, in [6] it is proved that:

$$
\lim _{h \rightarrow 0} \frac{1}{h^{2}} \inf E^{h}=0
$$

if and only if the following Riemann curvatures of $G$ vanish identically:

$$
R_{1212}=R_{1213}=R_{1223} \equiv 0 \quad \text { in } \Omega^{h} .
$$

More generally, the limit behavior of the rescaled energies $h^{-2} E^{h}$ has been investigated in [6] and it has been proved that their $\Gamma$-limit is given by the functional:

$$
\mathcal{I}_{2}(y)=\frac{1}{24} \int_{\Omega} \mathcal{Q}_{2, A}\left(x^{\prime},(\nabla y)^{t} \nabla \vec{b}\right) \mathrm{d} x^{\prime},
$$

effectively defined on the set of all $y \in W^{2,2}\left(\Omega, \mathbb{R}^{3}\right)$ such that $(\nabla y)^{t} \nabla y=G_{2 \times 2}$. The quadratic forms $\mathcal{Q}_{2, A}\left(x^{\prime}, \cdot\right)$ are given by means of the energy density $W$ as in (3.4). The Cosserat vector $\vec{b} \in W^{1,2} \cap L^{\infty}\left(\Omega, \mathbb{R}^{3}\right)$ is uniquely determined from the isometric immersion $y$ by:

$$
Q^{t} Q=G \quad \text { where } Q e_{1}=\partial_{1} y, \quad Q e_{2}=\partial_{2} y, \quad Q e_{3}=\vec{b}, \quad \text { with } \operatorname{det} Q>0 .
$$

Observe that the functional $\mathcal{I}_{2}$ is a Kirchhoff-like fully nonlinear bending energy, which in case of $G e_{3}=e_{3}$ reduces to the classical bending content quantifying the second fundamental form $(\nabla y)^{t} \nabla \vec{b}=(\nabla y)^{t} \nabla \vec{N}$ on the deformed surface $y(\Omega)$ with the unit normal vector $\vec{N}$. 
We recall that by Theorems 5.3, 5.5 and Corollary 5.4 in [6], the negation of condition (1.5) is equivalent to $\min \mathcal{I}_{2}>0$. For this reason, (1.5) is equivalent to the existence of a, necessarily unique and smooth, isometric immersion $y_{0}: \Omega \rightarrow \mathbb{R}^{3}$ of $G_{2 \times 2}$, such that:

$$
\left\{\begin{array}{l}
\left(\nabla y_{0}\right)^{t} \nabla y_{0}=G_{2 \times 2} \\
\operatorname{sym}\left(\left(\nabla y_{0}\right)^{t} \nabla \vec{b}_{0}\right)=0 .
\end{array}\right.
$$

Above, the smooth vector field $\vec{b}_{0}$ and the smooth matrix field $Q_{0}$ are given as in (1.6):

$$
Q_{0}^{t} Q_{0}=G, \quad Q_{0} e_{1}=\partial_{1} y_{0}, \quad Q_{0} e_{2}=\partial_{2} y_{0} \quad \text { and } Q_{0} e_{3}=\vec{b}_{0} \quad \text { with } \operatorname{det} Q_{0}>0 .
$$

Equivalently, denoting the inverse matrix $G^{-1}=\left[G^{i j}\right]_{i, j: 1 . .3}$, we have:

$$
\vec{b}_{0}=-\frac{1}{G^{33}}\left(G^{13} \partial_{1} y_{0}+G^{23} \partial_{2} y_{0}\right)+\frac{1}{\sqrt{G^{33}}} \vec{N} .
$$

Uniqueness of the immersion $y_{0}$ in (1.7) follows from Theorem 5.3 in [6] which shows that the second fundamental form of the surface $y_{0}(\Omega)$ is given in terms of $G$. Therefore, both fundamental forms are known. Also, the second equation in (1.7) comes from the fact that the kernel of each quadratic form $\mathcal{Q}_{2, A}$ consists of $s o(2)$.

1.4. New results of this work. In view of the above statements, in this paper we investigate smaller energy scalings and the limiting behaviour of the minimizing configurations to $E^{h}$ under condition (1.5). We first prove (in Lemma 2.1) that (1.5), which as we recall is equivalent to (1.1), implies:

$$
\inf E^{h} \leq C h^{4} .
$$

We then derive (in Theorem 3.1 and Theorem 4.1) the $\Gamma$-limit $\mathcal{I}_{4}$ of the rescaled energies $h^{-4} E^{h}$, together with their compactness properties.

Namely, let $y_{0}$ be the unique immersion satisfying (1.7), where $\vec{b}_{0}$ is as in (1.8). Let $\vec{d}_{0}: \Omega \rightarrow \mathbb{R}^{3}$ be the smooth vector field given in terms of $y_{0}$ by:

$$
\left\langle Q_{0}^{t} \vec{d}_{0}, e_{1}\right\rangle=-\left\langle\partial_{1} \vec{b}_{0}, \vec{b}_{0}\right\rangle, \quad\left\langle Q_{0}^{t} \vec{d}_{0}, e_{2}\right\rangle=-\left\langle\partial_{2} \vec{b}_{0}, \vec{b}_{0}\right\rangle, \quad\left\langle Q_{0}^{t} \vec{d}_{0}, e_{3}\right\rangle=0 .
$$

The limit $\mathcal{I}_{4}$ is then the following energy functional:

$$
\begin{aligned}
\mathcal{I}_{4}(V, \mathbb{S})= & \frac{1}{2} \int_{\Omega} \mathcal{Q}_{2, A}\left(x^{\prime}, \mathbb{S}+\frac{1}{2}(\nabla V)^{t} \nabla V+\frac{1}{24}\left(\nabla \vec{b}_{0}\right)^{t} \nabla \vec{b}_{0}\right) \mathrm{d} x^{\prime} \\
& +\frac{1}{24} \int_{\Omega} \mathcal{Q}_{2, A}\left(x^{\prime},\left(\nabla y_{0}\right)^{t} \nabla \vec{p}+(\nabla V)^{t} \nabla \vec{b}_{0}\right) \mathrm{d} x^{\prime} \\
& +\frac{1}{1440} \int_{\Omega} \mathcal{Q}_{2, A}\left(x^{\prime},\left(\nabla y_{0}\right)^{t} \nabla \vec{d}_{0}+\left(\nabla \vec{b}_{0}\right)^{t} \nabla \vec{b}_{0}\right) \mathrm{d} x^{\prime},
\end{aligned}
$$

acting on the space of finite strains:

$$
\mathbb{S} \in \operatorname{cl}_{L^{2}}\left\{\operatorname{sym}\left(\left(\nabla y_{0}\right)^{t} \nabla w\right) ; w \in W^{1,2}\left(\Omega, \mathbb{R}^{3}\right)\right\}
$$

and the space of first order infinitesimal isometries:

$$
V \in W^{2,2}\left(\Omega, \mathbb{R}^{3}\right) \quad \text { such that: } \quad \operatorname{sym}\left(\left(\nabla y_{0}\right)^{t} \nabla V\right)_{2 \times 2}=0,
$$


where the vector field $\vec{p} \in W^{1,2}\left(\Omega, \mathbb{R}^{3}\right)$ is uniquely associated with each $V$ by: $\left(\nabla y_{0}\right)^{t} \vec{p}=$ $-(\nabla V)^{t} \vec{b}_{0}$ and $\left\langle\vec{b}_{0}, \vec{p}\right\rangle=0$.

The spaces consisting of $\mathbb{S}$ and $V$ contain the information about the admissible error displacements, relative to the leading order immersion $y_{0}$, under the energy scaling $E^{h} \sim h^{4}$. We discuss the geometrical significance of $V$ and $\mathbb{S}$ and of various bending and stretching tensors in the first two terms of $\mathcal{I}_{4}(V, \mathbb{S})$ in section 5 . We further prove in Theorem 6.2 that the last term in (1.11), which is obviously constant and as such does not play a role in the minimization process, is precisely given by the only potentially nonzero (in view of (1.5)) curvatures of $G$, namely:

$$
\operatorname{sym}\left(\left(\nabla y_{0}\right)^{t} \nabla \vec{d}_{0}\right)+\left(\nabla \vec{b}_{0}\right)^{t} \nabla \vec{b}_{0}=\left[\begin{array}{ll}
R_{1313} & R_{1323} \\
R_{1323} & R_{2323}
\end{array}\right] .
$$

We may thus write, informally:

$$
\begin{gathered}
\mathcal{I}_{4}(V, \mathbb{S})=\frac{1}{2} \int_{\Omega} \mathcal{Q}_{2, A}\left(x^{\prime}, \text { stretching of order } h^{2}\right) \mathrm{d} x^{\prime}+\frac{1}{24} \int_{\Omega} \mathcal{Q}_{2, A}\left(x^{\prime}, \text { bending of order } h\right) \mathrm{d} x^{\prime} \\
+\frac{1}{1440} \int_{\Omega} \mathcal{Q}_{2, A}\left(x^{\prime}, \text { Riemann curvature of } G\right) \mathrm{d} x^{\prime} .
\end{gathered}
$$

In particular, since all three terms above are nonnegative, this directly implies that the condition $\lim _{h \rightarrow 0} \frac{1}{h^{4}}$ inf $E^{h}=0$, which is equivalent to $\min \mathcal{I}_{4}=0$, is further equivalent to the immersability $G$, i.e. the vanishing of all its Riemann curvatures $\operatorname{Riem}(G) \equiv 0$ in $\Omega^{h}$.

1.5. Acknowledgments. M.L. was partially supported by the NSF grant DMS-0846996 and the NSF grant DMS-1406730. A part of this work has been carried out while the first author visited the second author at the Université Paris Descartes, whose support and warm hospitality are gratefully acknowledged.

\section{The scaling and approximation lemmas}

We first introduce the following notation. Let $B_{0}\left(x^{\prime}\right)$ be the matrix field satisfying:

$$
B_{0} e_{1}=\partial_{1} \vec{b}_{0}, \quad B_{0} e_{2}=\partial_{2} \vec{b}_{0} \text { and } B_{0} e_{3}=\vec{d}_{0},
$$

where $\vec{d}_{0}$ is given by (1.10). Observe that in this way $Q_{0}^{t} B_{0}$ is skew symmetric. Indeed, it has the following block form:

$$
Q_{0}^{t} B_{0}=\left[\begin{array}{c|c}
\left(\nabla y_{0}\right)^{t} \nabla \vec{b}_{0} & \left(\nabla y_{0}\right)^{t} \vec{d}_{0} \\
\hline\left(\vec{b}_{0}\right)^{t} \nabla \vec{b}_{0} & \left\langle\vec{b}_{0}, \vec{d}_{0}\right\rangle
\end{array}\right]
$$

and by (1.7) we see that $\left(\nabla y_{0}\right)^{t} \nabla \vec{b}_{0} \in s o(2)$ is skew symmetric, while by (1.10): $\left(\nabla y_{0}\right)^{t} \vec{d}_{0}=$ $-\left(\nabla \vec{b}_{0}\right)^{t} \vec{b}_{0}$ and $\left\langle\vec{b}_{0}, \vec{d}_{0}\right\rangle=0$.

Lemma 2.1. Condition (1.5) implies: $\inf _{W^{1,2}\left(\Omega^{h}, \mathbb{R}^{3}\right)} E^{h} \leq C h^{4}$.

Proof. Let us construct a sequence $u^{h} \in W^{1,2}\left(\Omega^{h}, \mathbb{R}^{3}\right)$ that has low energy. Let:

$$
u^{h}\left(x^{\prime}, x_{3}\right)=y_{0}\left(x^{\prime}\right)+x_{3} \vec{b}_{0}\left(x^{\prime}\right)+\frac{x_{3}^{2}}{2} \vec{d}_{0}\left(x^{\prime}\right)
$$


in fact each $u^{h}$ is the restriction on its domain $\Omega^{h}$ of the same deformation. We have:

$$
\nabla u^{h}\left(x^{\prime}, x_{3}\right)=Q_{0}\left(x^{\prime}\right)+x_{3} B_{0}\left(x^{\prime}\right)+\frac{x_{3}^{2}}{2} D_{0}\left(x^{\prime}\right),
$$

where the matrix field $D_{0}\left(x^{\prime}\right) \in \mathbb{R}^{3 \times 3}$ is given through:

$$
D_{0}\left(x^{\prime}\right) e_{1}=\partial_{1} \vec{d}_{0}, \quad D_{0}\left(x^{\prime}\right) e_{2}=\partial_{2} \overrightarrow{d_{0}}, \quad D_{0}\left(x^{\prime}\right) e_{3}=0,
$$

so that:

$$
\nabla u^{h} A^{-1}=Q_{0} A^{-1}+x_{3} B_{0} A^{-1}+\frac{x_{3}^{2}}{2} D_{0} A^{-1} .
$$

For brevity, denote $F^{h}=\nabla u^{h} A^{-1}$. Obviously, $F^{h}$ decomposes as:

$$
F^{h}\left(x^{\prime}, x_{3}\right)=Q_{0} A^{-1}\left(x^{\prime}\right)\left(\operatorname{Id}_{3}+x_{3} S\left(x^{\prime}\right)+x_{3}^{2} T\left(x^{\prime}\right)\right)=\left(Q_{0} A^{-1}\left(x^{\prime}\right)\right) G^{h}\left(x^{\prime}, x_{3}\right)
$$

with $S=A^{-1} Q_{0}^{t} B_{0} A^{-1}, T=\frac{1}{2} A^{-1} Q_{0}^{t} D_{0} A^{-1}$ and $G^{h}=\operatorname{Id}_{3}+x_{3} S+x_{3}^{2} T$. Since $Q_{0} A^{-1} \in$ $S O(3)$ by construction, frame indifference implies that $W\left(F^{h}\right)=W\left(\left(\left(G^{h}\right)^{t} G^{h}\right)^{1 / 2}\right)$. Note that $S$ is skew symmetric, because $Q_{0}^{t} B_{0}$ is skew symmetric. Therefore, $\left(G^{h}\right)^{t} G^{h}$ and the expansion of its square root do not contain terms linear in $x_{3}$. Indeed, letting $K=T+T^{t}-S^{2}$ :

$$
\left(\left(G^{h}\right)^{t} G^{h}\right)\left(x^{\prime}, x_{3}\right)=\operatorname{Id}_{3}+x_{3}^{2} K\left(x^{\prime}\right)+\mathcal{O}\left(x_{3}^{3}\right)
$$

and:

$$
\left(\left(G^{h}\right)^{t} G^{h}\right)^{1 / 2}\left(x^{\prime}, x_{3}\right)=\operatorname{Id}_{3}+\frac{x_{3}^{2}}{2} K\left(x^{\prime}\right)+\mathcal{O}\left(x_{3}^{3}\right) .
$$

As a consequence, using $W\left(\operatorname{Id}_{3}\right)=0$ and $D W\left(\operatorname{Id}_{3}\right)=0$, we obtain:

$$
W\left(F^{h}\right)=W\left(\left(\left(G^{h}\right)^{t} G^{h}\right)^{1 / 2}\right)=\frac{x_{3}^{4}}{8} D^{2} W\left(\operatorname{Id}_{3}\right)(K, K)+\mathcal{O}\left(x_{3}^{5}\right) .
$$

Using (1.3), we get

$$
E^{h}\left(u^{h}\right)=\frac{1}{h} \int_{\Omega^{h}} W\left(F^{h}\right) \mathrm{d} x \leq C h^{4},
$$

which accomplishes the proof of the lemma.

In Lemma 2.1 above, we constructed deformations whose gradient was sufficiently close to $Q_{0}+x_{3} B_{0}$, to provide the energy of the order $h^{4}$. Conversely, in Corollary 2.3 below, we establish that the gradients of deformations $u^{h}$ whose energy scales like $h^{4}$, are close to $Q_{0}+x_{3} B_{0}$ modulo local multiplications by $R^{h}\left(x^{\prime}\right) \in S O(3)$. Corollary 2.3 makes this statement precise and gives an estimation on $\nabla R^{h}$ as well.

For any $\mathcal{V}$ which is an open subset of $\Omega$, we let $\mathcal{V}^{h}=\mathcal{V} \times(-h / 2, h / 2)$ and we define the local energy functional by:

$$
E^{h}\left(u^{h}, \mathcal{V}^{h}\right)=\frac{1}{h} \int_{\mathcal{V}^{h}} W\left(\nabla u^{h} A^{-1}\right) \mathrm{d} x .
$$


Lemma 2.2. Assume (1.5). There exists a constant $C>0$ with the following property. For any $u^{h} \in W^{1,2}\left(\mathcal{V}^{h}, \mathbb{R}^{3}\right)$, there exists $\bar{R}^{h} \in S O(3)$ such that:

$$
\frac{1}{h} \int_{\mathcal{V}^{h}}\left|\nabla u^{h}(x)-\bar{R}^{h}\left(Q_{0}\left(x^{\prime}\right)+x_{3} B_{0}\left(x^{\prime}\right)\right)\right|^{2} \mathrm{~d} x \leq C\left(E^{h}\left(u^{h}, \mathcal{V}^{h}\right)+h^{3}\left|\mathcal{V}^{h}\right|\right) .
$$

The constant $C$ is uniform for all $\mathcal{V}^{h}$ which are bi-Lipschitz equivalent with controlled Lipschitz constants.

Proof. By assumption (1.4), we have:

$$
E^{h}\left(u^{h}, \mathcal{V}^{h}\right) \geq \frac{c}{h} \int_{\mathcal{V}^{h}} \operatorname{dist}^{2}\left(\nabla u^{h} A^{-1}, S O(3)\right) \mathrm{d} x .
$$

This suggests performing a change of variables in order to use the nonlinear geometric rigidity estimate [29]. For any $u^{h} \in W^{1,2}\left(\mathcal{V}^{h}, \mathbb{R}^{3}\right)$, we let $v^{h}=u^{h} \circ Y^{-1}$ with $Y: \mathcal{V}^{h} \rightarrow Y\left(\mathcal{V}^{h}\right)=$ $\mathcal{U}^{h} \subset \mathbb{R}^{3}$ given as in (2.3), namely:

$$
Y\left(x^{\prime}, x_{3}\right)=y_{0}\left(x^{\prime}\right)+x_{3} \vec{b}_{0}\left(x^{\prime}\right)+\frac{x_{3}^{2}}{2} \vec{d}_{0}\left(x^{\prime}\right) .
$$

Obviously, $v^{h} \in W^{1,2}\left(\mathcal{U}^{h}, \mathbb{R}^{3}\right)$ and:

$$
\nabla u^{h} A^{-1}\left(x^{\prime}, x_{3}\right)=\nabla v^{h}(z)\left(\nabla Y A^{-1}\right)\left(x^{\prime}, x_{3}\right), \quad z:=Y\left(x^{\prime}, x_{3}\right) .
$$

Let $S^{\prime}=B_{0} Q_{0}^{-1}$ and $T^{\prime}=\frac{1}{2} D_{0} Q_{0}^{-1}$. Note that $S^{\prime}=B_{0} Q_{0}^{-1}=Q_{0}^{-1, t}\left(Q_{0}^{t} B_{0} Q_{0}^{-1}\right)=-Q_{0}^{-1, t} B_{0}^{t}$ in view of $Q_{0}^{t} B_{0} \in s o(3)$. Therefore $S^{\prime} \in s o(3)$. Computations as in Lemma 2.1 now give:

$$
\nabla Y\left(x^{\prime}, x_{3}\right)=Q_{0}\left(x^{\prime}\right)+x_{3} B_{0}\left(x^{\prime}\right)+\frac{x_{3}^{2}}{2} D_{0}\left(x^{\prime}\right),
$$

and:

$$
\nabla Y A^{-1}=\left(\operatorname{Id}_{3}+x_{3} S^{\prime}\left(x^{\prime}\right)+x_{3}^{2} T^{\prime}\left(x^{\prime}\right)\right)\left(Q_{0} A^{-1}\right) .
$$

We see that for $h$ small, $\operatorname{det}\left(\nabla Y A^{-1}\right)>0$. Further, the left polar decomposition $\nabla Y A^{-1}=$ $\left(\nabla Y A^{-1}\left(\nabla Y A^{-1}\right)^{t}\right)^{1 / 2} R$, allows us to write:

$$
\nabla Y A^{-1}=\left(\operatorname{Id}_{3}+x_{3}^{2} M\left(x^{\prime}, x_{3}\right)\right) R\left(x^{\prime}, x_{3}\right),
$$

where $M=\mathcal{O}(1)$ is a symmetric matrix field and $R \in S O(3)$. Again, the symmetric term does not contain any term linear in $x_{3}$. Therefore:

$$
\begin{aligned}
\operatorname{dist} & \left(\nabla v^{h} \nabla Y A^{-1}, S O(3)\right)=\operatorname{dist}\left(\nabla v^{h}\left(\operatorname{Id}_{3}+x_{3}^{2} M\right) R, S O(3)\right) \\
& =\operatorname{dist}\left(\nabla v^{h}\left(\operatorname{Id}_{3}+x_{3}^{2} M\right), S O(3)\right) \geq c \operatorname{dist}\left(\nabla v^{h}, S O(3)\left(\operatorname{Id}_{3}+x_{3}^{2} M\right)^{-1}\right) \\
& \geq c \operatorname{dist}\left(\nabla v^{h}, S O(3)\right)+\mathcal{O}\left(x_{3}^{2}\right) .
\end{aligned}
$$

Now, let $J=\left|\operatorname{det} \nabla Y \circ Y^{-1}\right|^{-1}$. By (2.7) and the above computation:

$$
\int_{\mathcal{V}^{h}} \operatorname{dist}^{2}\left(\nabla u^{h} A^{-1}, S O(3)\right) \mathrm{d} x \geq c \int_{\mathcal{U}^{h}} \operatorname{dist}^{2}\left(\nabla v^{h}, S O(3)\right) J \mathrm{~d} z-c \int_{\mathcal{V}^{h}} x_{3}^{4} \mathrm{~d} x .
$$


In other words, since $J \geq c>0$ :

$$
\frac{1}{h} \int_{\mathcal{V}^{h}} \operatorname{dist}^{2}\left(\nabla u^{h} A^{-1}, S O(3)\right) \mathrm{d} x+h^{3}\left|\mathcal{V}^{h}\right| \geq \frac{c}{h} \int_{\mathcal{U}^{h}} \operatorname{dist}^{2}\left(\nabla v^{h}, S O(3)\right) \mathrm{d} z .
$$

By [29], there exists $C>0$ with the following property. For any $v^{h} \in W^{1,2}\left(\mathcal{U}^{h}, \mathbb{R}^{3}\right)$, there exists $\bar{R}^{h} \in S O(3)$ such that:

$$
C \int_{\mathcal{U}^{h}} \operatorname{dist}^{2}\left(\nabla v^{h}, S O(3)\right) \mathrm{d} z \geq \int_{\mathcal{U}^{h}}\left|\nabla v^{h}-\bar{R}^{h}\right|^{2} \mathrm{~d} z .
$$

The constant $C$ can be chosen uniformly for domains $\mathcal{U}^{h}$ which are bi-Lipschitz equivalent with controlled Lipschitz constants. By (2.6) and the reverse change of variables which satisfies $J^{-1} \geq c>0$ and $|\nabla Y| \leq C$, we obtain:

$$
C\left(E^{h}\left(u^{h}, \mathcal{V}^{h}\right)+h^{3}\left|\mathcal{V}^{h}\right|\right) \geq \frac{1}{h} \int_{\mathcal{V}^{h}}\left|\nabla u^{h}-\bar{R}^{h} \nabla Y\right|^{2} \mathrm{~d} x
$$

again with a constant $C$ uniform for domains $\mathcal{V}^{h}$ that are bi-Lipschitz equivalent with controlled Lipschitz constants. This accomplishes the proof in view of (2.8).

Corollary 2.3. Assume (1.5) and let $u^{h}$ be a sequence of deformations such that:

$$
\lim _{h \rightarrow 0} h^{-2} E^{h}\left(u^{h}\right)=0 .
$$

Then, there exist matrix fields $R^{h} \in W^{1,2}(\Omega, S O(3))$ such that:

$$
\frac{1}{h} \int_{\Omega^{h}}\left|\nabla u^{h}(x)-R^{h}\left(x^{\prime}\right)\left(Q_{0}\left(x^{\prime}\right)+x_{3} B_{0}\left(x^{\prime}\right)\right)\right|^{2} \mathrm{~d} x \leq C\left(E^{h}\left(u^{h}\right)+h^{4}\right)
$$

and:

$$
\int_{\Omega}\left|\nabla R^{h}\left(x^{\prime}\right)\right|^{2} \mathrm{~d} x^{\prime} \leq \frac{C}{h^{2}}\left(E^{h}\left(u^{h}\right)+h^{4}\right) .
$$

The proof follows the lines of $[29,44,38]$, with necessary modifications in view of the expected error of the order $h^{4}$. For completeness, we will present the details in the Appendix.

\section{THE LOWER BOUND}

Theorem 3.1. Let $u^{h} \in W^{1,2}\left(\Omega^{h}, \mathbb{R}^{3}\right)$ be a sequence of deformations satisfying $E^{h}\left(u^{h}\right) \leq$ $C h^{4}$. Then there exists a sequence of translations $c^{h} \in \mathbb{R}^{3}$ and rotations $\bar{R}^{h} \in S O(3)$ such that the associated renormalizations:

$$
y^{h}\left(x^{\prime}, x_{3}\right)=\left(\bar{R}^{h}\right)^{t} u^{h}\left(x^{\prime}, h x_{3}\right)-c^{h} \in W^{1,2}\left(\Omega^{1}, \mathbb{R}^{3}\right)
$$

have the following properties, where $y_{0}$ and $\vec{b}_{0}$ are the unique solution to (1.7) (1.8). All convergences hold up to a subsequence:

(i) $y^{h} \rightarrow y_{0}$ in $W^{1,2}\left(\Omega^{1}, \mathbb{R}^{3}\right)$ and $\frac{1}{h} \partial_{3} y^{h} \rightarrow \vec{b}_{0}$ in $L^{2}\left(\Omega^{1}, \mathbb{R}^{3}\right)$; 
(ii) the scaled average displacements:

$$
V^{h}\left(x^{\prime}\right)=\frac{1}{h} f_{-\frac{1}{2}}^{-\frac{1}{2}}\left(y^{h}\left(x^{\prime}, x_{3}\right)-\left(y_{0}\left(x^{\prime}\right)+h x_{3} \vec{b}_{0}\left(x^{\prime}\right)\right)\right) \mathrm{d} x_{3}
$$

converge in $W^{1,2}\left(\Omega, \mathbb{R}^{3}\right)$ to a limiting field $V \in W^{2,2}\left(\Omega, \mathbb{R}^{3}\right)$, satisfying the constraint:

$$
\operatorname{sym}\left(\left(\nabla y_{0}\right)^{t} \nabla V\right)=0 ;
$$

(iii) the scaled tangential strains:

$$
\frac{1}{h} \operatorname{sym}\left(\left(\nabla y_{0}\right)^{t} \nabla V^{h}\right)
$$

converge weakly in $L^{2}\left(\Omega, \mathbb{R}^{2 \times 2}\right)$ to some $\mathbb{S} \in L^{2}\left(\Omega, \mathbb{R}_{s y m}^{2 \times 2}\right)$.

(iv) Further, defining the quadratic forms $\mathcal{Q}_{3}(F)=D^{2} W\left(\operatorname{Id}_{3}\right)(F, F)$ and:

$$
\mathcal{Q}_{2, A}\left(x^{\prime}, F_{2 \times 2}\right)=\min \left\{\mathcal{Q}_{3}\left(A\left(x^{\prime}\right)^{-1} \tilde{F} A\left(x^{\prime}\right)^{-1}\right) ; \tilde{F} \in \mathbb{R}^{3 \times 3} \text { with } \tilde{F}_{2 \times 2}=F_{2 \times 2}\right\},
$$

we have:

$$
\begin{aligned}
\liminf _{h \rightarrow 0} \frac{1}{h^{4}} E^{h}\left(u^{h}\right) \geq \mathcal{I}_{4}(V, \mathbb{S})= & \frac{1}{2} \int_{\Omega} \mathcal{Q}_{2, A}\left(x^{\prime}, \mathbb{S}+\frac{1}{2}(\nabla V)^{t} \nabla V+\frac{1}{24}\left(\nabla \vec{b}_{0}\right)^{t} \nabla \vec{b}_{0}\right) \mathrm{d} x^{\prime} \\
& +\frac{1}{24} \int_{\Omega} \mathcal{Q}_{2, A}\left(x^{\prime},\left(\nabla y_{0}\right)^{t} \nabla \vec{p}+(\nabla V)^{t} \nabla \vec{b}_{0}\right) \mathrm{d} x^{\prime} \\
& +\frac{1}{1440} \int_{\Omega} \mathcal{Q}_{2, A}\left(x^{\prime},\left(\nabla y_{0}\right)^{t} \nabla \vec{d}_{0}+\left(\nabla \vec{b}_{0}\right)^{t} \nabla \vec{b}_{0}\right) \mathrm{d} x^{\prime},
\end{aligned}
$$

where the vector field $\vec{p} \in W^{1,2}\left(\Omega, \mathbb{R}^{3}\right)$ is uniquely associated with $V$ by:

$$
\left\{\begin{array}{l}
\left(\nabla y_{0}\right)^{t} \vec{p}=-(\nabla V)^{t} \vec{b}_{0} \\
\left\langle\vec{b}_{0}, \vec{p}\right\rangle=0
\end{array}\right.
$$

Proof. 1. Corollary 2.3 yields existence of $R^{h} \in W^{1,2}(\Omega, S O(3))$ such that (2.9) and (2.10) hold with $C h^{4}$ and $C h^{2}$ in their right hand sides, respectively. We rewrite these inequalities for the reader's convenience:

$$
\frac{1}{h} \int_{\Omega^{h}}\left|\nabla u^{h}(x)-R^{h}\left(x^{\prime}\right)\left(Q_{0}\left(x^{\prime}\right)+x_{3} B_{0}\left(x^{\prime}\right)\right)\right|^{2} \mathrm{~d} x \leq C h^{4}
$$

and:

$$
\int_{\Omega}\left|\nabla R^{h}\left(x^{\prime}\right)\right|^{2} \mathrm{~d} x^{\prime} \leq C h^{2}
$$

To prove the claimed convergence properties for (3.1), it is natural in view of (3.7) to set:

$$
\bar{R}^{h}=\mathbb{P}_{\mathrm{SO}(3)} f_{\Omega^{h}} \nabla u^{h}(x) Q_{0}\left(x^{\prime}\right)^{-1} \mathrm{~d} x .
$$


This projection is well defined, because for every $x^{\prime} \in \Omega$, in view of (3.7):

$$
\begin{aligned}
\operatorname{dist}^{2}\left(f_{\Omega^{h}} \nabla u^{h}\right. & \left.Q_{0}^{-1} \mathrm{~d} x, S O(3)\right) \leq\left|f_{\Omega^{h}} \nabla u^{h} Q_{0}^{-1} \mathrm{~d} x-R^{h}\left(x^{\prime}\right)\right|^{2} \\
& \leq C\left|f_{\Omega^{h}}\left(\nabla u^{h} Q_{0}^{-1}-R^{h}\right) \mathrm{d} x\right|^{2}+C\left|f_{\Omega^{h}} R^{h} \mathrm{~d} x-R^{h}\left(x^{\prime}\right)\right|^{2} \\
& \leq C\left|f_{\Omega^{h}}\left(\nabla u^{h}-R^{h}\left(Q_{0}+x_{3} B_{0}\right)\right) Q_{0}^{-1}\right|^{2} \mathrm{~d} x+C\left|R^{h}\left(x^{\prime}\right)-f_{\Omega} R^{h}\right|^{2} \\
& \leq C f_{\Omega^{h}}\left|\nabla u^{h}-R^{h}\left(Q_{0}+x_{3} B_{0}\right)\right|^{2} \mathrm{~d} x+C\left|R^{h}\left(x^{\prime}\right)-f_{\Omega} R^{h}\right|^{2} \\
& \leq C h^{4}+C\left|R^{h}\left(x^{\prime}\right)-f_{\Omega} R^{h}\right|^{2}
\end{aligned}
$$

Now, taking the average on $\Omega$, by the Poincaré-Wirtinger inequality and (3.8), we get:

$$
\operatorname{dist}^{2}\left(f_{\Omega^{h}} \nabla u^{h} Q_{0}^{-1} \mathrm{~d} x, S O(3)\right) \leq C h^{4}+C \int_{\Omega}\left|\nabla R^{h}\right|^{2} \leq C h^{2},
$$

which proves that the average $f_{\Omega^{h}} \nabla u^{h} Q_{0}^{-1} \mathrm{~d} x$ is close to $S O(3)$ and that:

$$
\left|f_{\Omega^{h}} \nabla u^{h} Q_{0}^{-1} \mathrm{~d} x-\bar{R}^{h}\right|^{2} \leq C h^{2} .
$$

Moreover:

$$
\begin{aligned}
& f_{\Omega}\left|R^{h}-\bar{R}^{h}\right|^{2} \mathrm{~d} x=f_{\Omega^{h}}\left|R^{h}-\bar{R}^{h}\right|^{2} \mathrm{~d} x \\
& \leq C f_{\Omega^{h}}\left(\left|R^{h}-f_{\Omega} R^{h}\right|^{2}+\left|\left(f_{\Omega} R^{h}\right)-f_{\Omega^{h}} \nabla u^{h} Q_{0}^{-1}\right|^{2}\right)+f_{\Omega^{h}}\left|\bar{R}^{h}-f_{\Omega^{h}} \nabla u^{h} Q_{0}^{-1}\right|^{2} \\
& \leq C f_{\Omega^{h}}\left|\nabla R^{h}\right|^{2} \mathrm{~d} x+C f_{\Omega^{h}}\left|\nabla u^{h}-R^{h}\left(Q_{0}+x_{3} B_{0}\right)\right|^{2} \mathrm{~d} x+C h^{2} \leq C h^{2}
\end{aligned}
$$

where the last estimate follows by (3.7), (3.8) and (3.9).

Let now $c^{h} \in \mathbb{R}^{3}$ be such that $\int_{\Omega} V^{h}=0$ where $V^{h}$ is defined as in (3.2). Denote by $\nabla_{h} y^{h}$ the matrix whose columns are given by $\partial_{1} y^{h}, \partial_{2} y^{h}$ and $\partial_{3} y^{h} / h$. Obviously:

$$
\nabla_{h} y^{h}\left(x^{\prime}, x_{3}\right)=\left(\bar{R}^{h}\right)^{t} \nabla u^{h}\left(x^{\prime}, h x_{3}\right) .
$$

Observe that:

$$
\begin{aligned}
& \int_{\Omega^{1}}\left|\nabla_{h} y^{h}-Q_{0}\right|^{2} \mathrm{~d} x \leq C f_{\Omega^{h}}\left|\nabla u^{h}-\bar{R}^{h} Q_{0}\right|^{2} \mathrm{~d} x \\
& \quad \leq C\left(f_{\Omega^{h}}\left|\nabla u^{h}-R^{h}\left(Q_{0}+x_{3} B_{0}\right)\right|^{2} \mathrm{~d} x+f_{\Omega^{h}}\left|x_{3} R^{h} B_{0}\right|^{2} \mathrm{~d} x+f_{\Omega^{h}}\left|R^{h}-\bar{R}^{h}\right|^{2} \mathrm{~d} x\right) \leq C h^{2}
\end{aligned}
$$

by (3.7) and (3.10). Therefore, $\nabla_{h} y^{h}$ converges in $L^{2}\left(\Omega^{1}\right)$ to $Q_{0}$. Further, the sequence $\left\{y^{h}\right\}$ is bounded in $W^{1,2}\left(\Omega^{1}\right)$, by the choice of $c^{h}$. Passing to a subsequence we get that $y^{h}$ 
converges weakly in $W^{1,2}\left(\Omega^{1}\right)$ and in view of the strong convergence of $\nabla y^{h}$ we have:

$$
y^{h} \rightarrow y_{0} \quad \text { in } \quad W^{1,2}\left(\Omega^{1}, \mathbb{R}^{3}\right) \quad \text { and } \quad \frac{1}{h} \partial_{3} y^{h} \rightarrow \vec{b}_{0} \quad \text { in } \quad L^{2}\left(\Omega^{1}, \mathbb{R}^{3}\right) .
$$

2. Note that, for every $x^{\prime} \in \Omega$ :

$$
\begin{aligned}
& \nabla V^{h}\left(x^{\prime}\right)=\frac{1}{h}\left(f_{-1 / 2}^{1 / 2} \nabla_{h} y^{h}(x)-Q_{0}\left(x^{\prime}\right) \mathrm{d} x_{3}\right)_{3 \times 2} \\
& =\frac{1}{h}\left(f_{-1 / 2}^{1 / 2} \nabla_{h} y^{h}-\left(\bar{R}^{h}\right)^{t} R^{h}\left(Q_{0}+h x_{3} B_{0}\right) \mathrm{d} x_{3}\right)_{3 \times 2}+\frac{1}{h}\left(\left(\left(\bar{R}^{h}\right)^{t} R^{h}-\operatorname{Id}_{3}\right) Q_{0}\right)_{3 \times 2} \\
& =I_{1}^{h}+I_{2}^{h} .
\end{aligned}
$$

The first term above converges to 0 . Indeed:

$$
\begin{aligned}
\left\|I_{1}^{h}\right\|_{L^{2}(\Omega)}^{2} & \leq \frac{C}{h^{2}} f_{\Omega^{1}}\left|\left(\bar{R}^{h}\right)^{t} \nabla u^{h}\left(x^{\prime}, h x_{3}\right)-\left(\bar{R}^{h}\right)^{t} R^{h}\left(Q_{0}\left(x^{\prime}\right)+h x_{3} B_{0}\right)\right|^{2} \mathrm{~d} x \\
& \leq \frac{C}{h^{2}} f_{\Omega^{h}}\left|\nabla u^{h}\left(x^{\prime}, x_{3}\right)-R^{h}\left(Q_{0}+x_{3} B_{0}\right)\right|^{2} \mathrm{~d} x \leq C h^{2} .
\end{aligned}
$$

Towards estimating the second term in (3.12), denote:

$$
S^{h}=\frac{1}{h}\left(\left(\bar{R}^{h}\right)^{t} R^{h}-\mathrm{Id}_{3}\right) .
$$

By (3.10) and (3.8), it follows that:

$$
\left\|S^{h}\right\|_{L^{2}(\Omega)}^{2} \leq \frac{C}{h^{2}} \int_{\Omega}\left|R^{h}-\bar{R}^{h}\right|^{2} \leq C \quad \text { and } \quad\left\|\nabla S^{h}\right\|_{L^{2}(\Omega)}^{2} \leq \frac{C}{h^{2}} \int_{\Omega}\left|\nabla R^{h}\right|^{2} \leq C .
$$

Passing to a subsequence, we can assume that:

$$
S^{h} \rightarrow S \quad \text { weakly in } W^{1,2}(\Omega),
$$

which implies:

$$
I_{2}^{h} \rightarrow\left(S Q_{0}\right)_{3 \times 2} \quad \text { in } L^{2}\left(\Omega, \mathbb{R}^{3 \times 2}\right) .
$$

Consequently, by (3.12):

$$
\nabla V^{h} \rightarrow\left(S Q_{0}\right)_{3 \times 2} \quad \text { in } L^{2}\left(\Omega, \mathbb{R}^{3 \times 2}\right) .
$$

As before, we conclude that $V^{h}$ converges in $W^{1,2}(\Omega)$ and that its limit $V$ belongs to $W^{2,2}\left(\Omega, \mathbb{R}^{3}\right)$, since $\nabla V=\left(S Q_{0}\right)_{3 \times 2} \in W^{1,2}(\Omega)$. We now prove (3.3). By definition of $S^{h}$ :

$$
\operatorname{sym} S^{h}=-\frac{h}{2}\left(S^{h}\right)^{t} S^{h},
$$

so in view of the boundedness of $\left\{S^{h}\right\}$ in $W^{1,2}$ :

$$
\left\|\operatorname{sym} S^{h}\right\|_{L^{2}(\Omega)} \leq C h\left\|S^{h}\right\|_{L^{4}(\Omega)}^{2} \leq C h\left\|S^{h}\right\|_{W^{1,2}(\Omega)}^{2} \leq C h .
$$

Consequently, $S$ is a skew symmetric field. But $\left(\nabla y_{0}\right)^{t} \nabla V=\left(Q_{0}^{t} S Q_{0}\right)_{2 \times 2}$, hence (3.3) follows. 
For future use, let us define $\vec{p} \in W^{1,2}\left(\Omega, \mathbb{R}^{3}\right)$ by:

$$
[\nabla V \mid \vec{p}]=S Q_{0} .
$$

Since $Q_{0}^{t}[\nabla V \mid p] \in s o(3)$, it is easily checked that $\vec{p}$ is given solely in terms of $V$ by:

$$
\left\{\begin{array}{l}
\left(\nabla y_{0}\right)^{t} \vec{p}=-(\nabla V)^{t} \vec{b}_{0} \\
\left\langle\vec{b}_{0}, \vec{p}\right\rangle=0
\end{array}\right.
$$

3. We now want to establish convergence in (iii). In view of (3.12) we write:

$$
\begin{aligned}
\frac{1}{h} \operatorname{sym}\left(Q_{0}^{t} \nabla V^{h}\right)_{2 \times 2}\left(x^{\prime}\right) & =\frac{1}{h} \operatorname{sym}\left(Q_{0}^{t} I_{1}^{h}\right)_{2 \times 2}+\frac{1}{h} \operatorname{sym}\left(Q_{0}^{t} S^{h} Q_{0}\right)_{2 \times 2} \\
& =J_{1}^{h}+J_{2}^{h} .
\end{aligned}
$$

We first deal with the sequence $J_{2}^{h}$. By (3.14), $S^{h} \rightarrow S$ in $L^{4}(\Omega)$ and so (3.17) implies:

$$
\frac{1}{h} \operatorname{sym} S^{h} \rightarrow-\frac{1}{2} S^{t} S=\frac{1}{2} S^{2} \quad \text { in } L^{2}(\Omega) .
$$

Therefore:

$$
J_{2}^{h} \rightarrow-\frac{1}{2}\left(Q_{0}^{t} S^{t} S Q_{0}\right)_{2 \times 2}=-\frac{1}{2}(\nabla V)^{t} \nabla V \quad \text { in } \quad L^{2}(\Omega) .
$$

We now prove that $J_{1}^{h}$ converges. Recall that by (3.20), (3.12) and (3.11):

$$
J_{1}^{h}=\frac{1}{h} \operatorname{sym}\left(Q_{0}^{t} I_{1}^{h}\right)_{2 \times 2}=\operatorname{sym}\left(Q_{0}^{t}\left(\bar{R}^{h}\right)^{t} f_{-1 / 2}^{1 / 2} Z^{h}\left(x^{\prime}, x_{3}\right) \mathrm{d} x_{3}\right)_{2 \times 2}
$$

where the rescaled strains $Z^{h}$ are defined by:

$$
Z^{h}\left(x^{\prime}, x_{3}\right)=\frac{1}{h^{2}}\left(\nabla u^{h}\left(x^{\prime}, h x_{3}\right)-R^{h}\left(x^{\prime}\right)\left(Q_{0}\left(x^{\prime}\right)+h x_{3} B_{0}\left(x^{\prime}\right)\right)\right) .
$$

By (3.7), the sequence $\left\{Z^{h}\right\}$ is bounded in $L^{2}\left(\Omega^{1}, \mathbb{R}^{3}\right)$. Therefore, up to a subsequence:

$$
Z^{h} \rightarrow Z \quad \text { weakly in } L^{2}\left(\Omega^{1}, \mathbb{R}^{3}\right) .
$$

It follows that:

$$
J_{1}^{h} \rightarrow J_{1}:=\operatorname{sym}\left(Q_{0}^{t}(\bar{R})^{t} f_{-1 / 2}^{1 / 2} Z\left(x^{\prime}, x_{3}\right) \mathrm{d} x_{3}\right)_{2 \times 2} \quad \text { weakly in } L^{2}(\Omega) .
$$

which yields (iii) by (3.20) and (3.21).

4. We now aim at giving the structure of the weak limit $\mathbb{S}$ of $\frac{1}{h} \operatorname{sym}\left(Q_{0}^{t} \nabla V^{h}\right)_{2 \times 2}$ in terms of the limiting fields $V$ and $Z$. We have just seen that:

$$
\mathbb{S}=J_{1}-\frac{1}{2}(\nabla V)^{t} \nabla V
$$

where $J_{1}$ is given by (3.25). As a tool, consider the difference quotients $f^{s, h}$ :

$$
f^{s, h}\left(x^{\prime}, x_{3}\right)=\frac{1}{h^{2} s}\left(y^{h}\left(x^{\prime}, x_{3}+s\right)-y^{h}\left(x^{\prime}, x_{3}\right)-h s\left(\vec{b}_{0}+h\left(x_{3}+\frac{s}{2}\right) \vec{d}_{0}\right)\right),
$$


and let us study for any $s$ the convergence of $f^{s, h}$ as $h \rightarrow 0$. In fact, we will show that $f^{s, h} \rightarrow \vec{p}$, weakly in $W^{1,2}\left(\Omega^{1}, \mathbb{R}^{3}\right)$. Write:

$$
f^{s, h}\left(x^{\prime}, x_{3}\right)=\frac{1}{h^{2}} f_{0}^{s} \partial_{3} y^{h}\left(x^{\prime}, x_{3}+t\right)-h\left(\vec{b}_{0}+h\left(x_{3}+t\right) \vec{d}_{0}\right) \mathrm{d} t,
$$

and observe that:

$$
\begin{aligned}
& \frac{1}{h^{2}}\left(\partial_{3} y^{h}-h\left(\vec{b}_{0}+h x_{3} \vec{d}_{0}\right)\right)=\frac{1}{h}\left(\left(\bar{R}^{h}\right)^{t} \nabla u^{h}\left(x^{\prime}, h x_{3}\right)-\left(Q_{0}+h x_{3} B_{0}\right)\right) e_{3} \\
& \quad=\frac{1}{h}\left(\bar{R}^{h}\right)^{t}\left(\nabla u^{h}\left(x^{\prime}, h x_{3}\right)-R^{h}\left(Q_{0}+h x_{3} B_{0}\right)\right) e_{3}+S^{h}\left(Q_{0}+h x_{3} B_{0}\right) e_{3} \\
& \quad=h\left(\bar{R}^{h}\right)^{t} Z^{h}\left(x^{\prime}, x_{3}\right) e_{3}+S^{h}\left(Q_{0}+h x_{3} B_{0}\right) e_{3} .
\end{aligned}
$$

The first term in the right hand side above converges to 0 in $L^{2}\left(\Omega^{1}\right)$ because $\left\{Z^{h}\right\}$ is bounded in $L^{2}\left(\Omega^{1}, \mathbb{R}^{3}\right)$, while the second term converges to $S Q_{0} e_{3}=S \vec{b}_{0}$ in $L^{2}\left(\Omega^{1}\right)$ by $(3.14)$. Note that $S Q_{0} e_{3}=\vec{p}$ by (3.18). Therefore, $f^{s, h} \rightarrow \vec{p}$ in $L^{2}\left(\Omega^{1}\right)$.

We now deal with the derivatives of the studied sequence. Firstly:

$$
\begin{array}{r}
\partial_{3} f^{s, h}\left(x^{\prime}, x_{3}\right)=\frac{1}{s}\left(\frac{1}{h^{2}}\left(\partial_{3} y^{h}\left(x^{\prime}, x_{3}+s\right)-h\left(\vec{b}_{0}+h\left(x_{3}+s\right) \vec{d}_{0}\right)\right)\right. \\
\left.-\frac{1}{h^{2}}\left(\partial_{3} y^{h}\left(x^{\prime}, x_{3}\right)-h\left(\vec{b}_{0}+h x_{3} \vec{d}_{0}\right)\right)\right)
\end{array}
$$

converges to 0 in $L^{2}\left(\Omega^{1}\right)$. For $i=1,2$, the in-plane derivatives read as:

$$
\begin{aligned}
\partial_{i} f^{s, h}\left(x^{\prime}, x_{3}\right)= & \frac{1}{h^{2} s}\left(\left(\bar{R}^{h}\right)^{t} \partial_{i} u^{h}\left(x^{\prime}, h\left(x_{3}+s\right)\right)\right. \\
& \left.\quad-\left(\bar{R}^{h}\right)^{t} \partial_{i} u^{h}\left(x^{\prime}, h x_{3}\right)-h s\left(\partial_{i} \vec{b}_{0}+h\left(x_{3}+\frac{s}{2}\right)\right) \partial_{i} \vec{d}_{0}\right) \\
= & \frac{1}{s}\left(\left(\bar{R}^{h}\right)^{t} Z^{h}\left(x^{\prime}, x_{3}+s\right)-\left(\overline{R^{h}}\right)^{t} Z^{h}\left(x^{\prime}, x_{3}\right)\right) e_{i} \\
& +\frac{1}{h^{2} s}\left(\left(\bar{R}^{h}\right)^{t} R^{h}\left(Q_{0}+h\left(x_{3}+s\right) B_{0}\right)-\left(\bar{R}^{h}\right)^{t} R^{h}\left(Q_{0}+h x_{3} B_{0}\right)\right) e_{i} \\
& -\frac{1}{h}\left(B_{0} e_{i}+h\left(x_{3}+\frac{s}{2}\right) \partial_{i} \vec{d}_{0}\right) .
\end{aligned}
$$

The last two terms above can be written as: $S^{h} B_{0} e_{i}-\left(x_{3}+\frac{s}{2}\right) \partial_{i} \vec{d}_{0}$, hence by (3.24):

$$
\begin{aligned}
\partial_{i} f^{s, h}\left(x^{\prime}, x_{3}\right) \rightarrow \frac{1}{s}(\bar{R})^{t}(Z & \left.\left(x^{\prime}, x_{3}+s\right)-Z\left(x^{\prime}, x_{3}\right)\right) e_{i} \\
+ & S B_{0} e_{i}-\left(x_{3}+\frac{s}{2}\right) \partial_{i} \vec{d}_{0} \quad \text { weakly in } L^{2}\left(\Omega^{1}, \mathbb{R}^{3}\right),
\end{aligned}
$$

where $\bar{R} \in S O(3)$ is an accumulation point of the rotations $\bar{R}^{h}$.

Consequently, $f^{s, h} \rightarrow \vec{p}$ weakly in $W^{1,2}\left(\Omega^{1}, \mathbb{R}^{3}\right)$ and, for $i=1,2$ :

$$
s \partial_{i} \vec{p}=(\bar{R})^{t}\left(Z\left(x^{\prime}, x_{3}+s\right)-Z\left(x^{\prime}, x_{3}\right)\right) e_{i}+s S B_{0} e_{i}-s\left(x_{3}+\frac{s}{2}\right) \partial_{i} \vec{d}_{0},
$$


which proves that $Z\left(x^{\prime}, \cdot\right) e_{i}$ has polynomial form and that:

$$
\left(\bar{R}^{t} Z\left(x^{\prime}, x_{3}\right)\right)_{3 \times 2}=\left(\bar{R}^{t} Z\left(x^{\prime}, 0\right)\right)_{3 \times 2}+x_{3}\left(\nabla \vec{p}-\left(S B_{0}\right)_{3 \times 2}\right)+\frac{x_{3}^{2}}{2} \nabla \overrightarrow{d_{0}} .
$$

By (3.24), it follows that:

$$
J_{1}=\operatorname{sym}\left(Q_{0}^{t}(\bar{R})^{t} Z\left(x^{\prime}, 0\right)\right)_{2 \times 2}+\frac{1}{24} \operatorname{sym}\left(Q_{0}^{t} \nabla \overrightarrow{d_{0}}\right)_{2 \times 2} .
$$

With (3.26), we finally arrive at the following identity that links $\mathbb{S}$ and $V$ and $Z$ :

$$
\mathbb{S}\left(x^{\prime}\right)=\operatorname{sym}\left(Q_{0}^{t}(\bar{R})^{t} Z\left(x^{\prime}, 0\right)\right)_{2 \times 2}+\frac{1}{24} \operatorname{sym}\left(Q_{0}^{t} \nabla \overrightarrow{d_{0}}\right)_{2 \times 2}-\frac{1}{2}(\nabla V)^{t} \nabla V .
$$

5. We now prove the lower bound in (iv). Recall that by (3.23):

$$
\nabla u^{h}\left(x^{\prime}, h x_{3}\right)=R^{h}\left(x^{\prime}\right)\left(Q_{0}\left(x^{\prime}\right)+h x_{3} B_{0}\left(x^{\prime}\right)\right)+h^{2} Z^{h}\left(x^{\prime}, x_{3}\right) .
$$

Since $Q_{0} A^{-1} \in S O(3)$ we have:

$$
W\left(\nabla u^{h} A^{-1}\right)=W\left(\left(Q_{0} A^{-1}\right)^{t}\left(R^{h}\right)^{t} \nabla u^{h} A^{-1}\right)=W\left(\operatorname{Id}_{3}+h \mathcal{J}+h^{2} \mathcal{G}^{h}\right),
$$

where:

$$
\mathcal{J}\left(x^{\prime}, x_{3}\right)=x_{3} A^{-1}\left(Q_{0}^{t} B_{0}\right) A^{-1}\left(x^{\prime}\right) \in \operatorname{so}(3), \quad \mathcal{G}^{h}\left(x^{\prime}, x_{3}\right)=A^{-1} Q_{0}^{t}\left(R^{h}\right)^{t} Z^{h}\left(x^{\prime}, x_{3}\right) A^{-1} .
$$

Note that by (3.24):

$$
\mathcal{G}^{h}\left(x^{\prime}, x_{3}\right) \rightarrow \mathcal{G}=A^{-1} Q_{0}^{t}\left(\bar{R}^{t}\right) Z\left(x^{\prime}, x_{3}\right) A^{-1} \quad \text { weakly in } L^{2}\left(\Omega^{1}, \mathbb{R}^{3 \times 3}\right) .
$$

Define the "good sets":

$$
\Omega_{h}=\left\{x \in \Omega^{1} ; h\left|\mathcal{G}^{h}\right|<1\right\} .
$$

By the above, the characteristic functions $\mathbb{1}_{\Omega_{h}}$ converge to $\mathbb{1}$ in $L^{1}(\Omega)$. Further, by frame invariance and Taylor expanding $W$ on $\Omega_{h}$ :

$$
\begin{aligned}
W\left(\operatorname{Id}_{3}+h \mathcal{J}+h^{2} \mathcal{G}^{h}\right) & =W\left(e^{-h \mathcal{J}}\left(\operatorname{Id}_{3}+h \mathcal{J}+h^{2} \mathcal{G}^{h}\right)\right) \\
& =W\left(\operatorname{Id}_{3}+h^{2}\left(\mathcal{G}^{h}-\frac{1}{2} \mathcal{J}^{2}\right)+o\left(h^{2}\right)\right) \\
& =\frac{1}{2} \mathcal{Q}_{3}\left(h^{2}\left(\mathcal{G}^{h}-\frac{1}{2} \mathcal{J}^{2}\right)\right)+o\left(h^{4}\right) .
\end{aligned}
$$

Therefore:

$$
\begin{aligned}
\liminf _{h \rightarrow 0} \frac{1}{h^{4}} E^{h}\left(u^{h}\right) & \geq \liminf _{h \rightarrow 0} \frac{1}{h^{4}} \int_{\Omega^{1}} \mathbb{1}_{\Omega_{h}} W\left(\operatorname{Id}_{3}+h \mathcal{J}+h^{2} \mathcal{G}^{h}\right) \mathrm{d} x \\
& =\liminf _{h \rightarrow 0} \frac{1}{2} \int_{\Omega^{1}} \mathcal{Q}_{3}\left(\mathbb{1}_{\Omega_{h}} \operatorname{sym}\left(\mathcal{G}^{h}-\frac{1}{2} \mathcal{J}^{2}\right)\right) \mathrm{d} x \\
& \geq \frac{1}{2} \int_{\Omega^{1}} \mathcal{Q}_{3}\left(\operatorname{sym}\left(\mathcal{G}-\frac{1}{2} \mathcal{J}^{2}\right)\right) \mathrm{d} x,
\end{aligned}
$$

by the weak sequential lower semi-continuity of the quadratic form $\mathcal{Q}_{3}$ in $L^{2}$ and in view of: $\mathbb{1}_{\Omega^{h} \operatorname{sym}}\left(\mathcal{G}^{h}-\frac{1}{2} \mathcal{J}^{2}\right) \rightarrow \operatorname{sym} \mathcal{G}-\frac{1}{2} \mathcal{J}^{2} \quad$ weakly in $L^{2}\left(\Omega^{1}\right)$. 
Note that by (3.18) we have: $\left(Q_{0}^{t} S B_{0}\right)_{2 \times 2}=-(\nabla V)^{t} \nabla \vec{b}_{0}$ and that:

$$
\mathcal{J}^{2}=-\mathcal{J}^{t} \mathcal{J}=-x_{3}^{2} A^{-1} B_{0}^{t} B_{0} A^{-1} .
$$

Therefore, using (3.28), the right hand side of (3.30) is bounded below by:

$$
\begin{gathered}
\frac{1}{2} \int_{\Omega^{1}} \mathcal{Q}_{2, A}\left(x^{\prime}, \operatorname{sym}\left(Q_{0}^{t}(\bar{R})^{t} Z\left(x^{\prime}, 0\right)+x_{3}\left(Q_{0}^{t} \nabla \vec{p}+(\nabla V)^{t} \nabla \vec{b}_{0}\right)+\frac{x_{3}^{2}}{2}\left(Q_{0}^{t} \nabla \vec{d}_{0}+\left(\nabla \vec{b}_{0}\right)^{t} \nabla \vec{b}_{0}\right)\right)_{2 \times 2}\right) \mathrm{d} x \\
=\frac{1}{2} \int_{\Omega^{1}} \mathcal{Q}_{2, A}\left(x^{\prime}, I\left(x^{\prime}\right)+x_{3} I I I\left(x^{\prime}\right)+x_{3}^{2} I I\left(x^{\prime}\right)\right) \mathrm{d} x .
\end{gathered}
$$

Above we used (3.29) and we denoted:

$$
\begin{aligned}
I\left(x^{\prime}\right) & =\mathbb{S}-\frac{1}{24} \operatorname{sym}\left(\left(\nabla y_{0}\right)^{t} \nabla \vec{d}_{0}\right)+\frac{1}{2}(\nabla V)^{t} \nabla V \\
I I\left(x^{\prime}\right) & =\frac{1}{2} \operatorname{sym}\left(\left(\nabla y_{0}\right)^{t} \nabla \vec{d}_{0}\right)+\frac{1}{2}\left(\nabla \vec{b}_{0}\right)^{t} \nabla \vec{b}_{0} \\
I I I\left(x^{\prime}\right) & =\operatorname{sym}\left(\left(\nabla y_{0}\right)^{t} \nabla \vec{p}\right)+\operatorname{sym}\left((\nabla V)^{t} \nabla \vec{b}_{0}\right) .
\end{aligned}
$$

Let $\mathcal{L}_{2, A}\left(x^{\prime}\right)$ be the symmetric bilinear form generating the quadratic form $\mathcal{Q}_{2, A}\left(x^{\prime}\right)$. Since the odd powers of $x_{3}$ integrate to 0 on the symmetric interval $(-1 / 2,1 / 2)$, we get:

$$
\begin{aligned}
& \int_{\Omega^{1}} \mathcal{Q}_{2, A}\left(x^{\prime}, I\left(x^{\prime}\right)+x_{3} I I I\left(x^{\prime}\right)+x_{3}^{2} I I\left(x^{\prime}\right)\right) \mathrm{d} x \\
&=\int_{\Omega} \mathcal{Q}_{2, A}\left(x^{\prime}, I\left(x^{\prime}\right)\right) \mathrm{d} x^{\prime}+\left(\int_{-1 / 2}^{1 / 2} x_{3}^{2} \mathrm{~d} x_{3}\right) \int_{\Omega} Q_{2, A}\left(x^{\prime}, I I I\left(x^{\prime}\right)\right) \mathrm{d} x^{\prime} \\
&+\left(\int_{-1 / 2}^{1 / 2} x_{3}^{4} \mathrm{~d} x_{3}\right) \int_{\Omega} Q_{2, A}\left(x^{\prime}, I I\left(x^{\prime}\right)\right) \mathrm{d} x^{\prime}+2\left(\int_{-1 / 2}^{1 / 2} x_{3}^{2} \mathrm{~d} x_{3}\right) \int_{\Omega} \mathcal{L}_{2, A}\left(x^{\prime}, I\left(x^{\prime}\right), I I\left(x^{\prime}\right)\right) \mathrm{d} x^{\prime} \\
&= \int_{\Omega} \mathcal{Q}_{2, A}\left(x^{\prime}, I\right)+\frac{1}{12} \int_{\Omega} Q_{2, A}\left(x^{\prime}, I I I\right)+\frac{1}{80} \int_{\Omega} Q_{2, A}\left(x^{\prime}, I I\right)+\frac{2}{12} \int_{\Omega} \mathcal{L}_{2, A}\left(x^{\prime}, I, I I\right) \mathrm{d} x^{\prime} \\
&= \int_{\Omega} \mathcal{Q}_{2, A}\left(x^{\prime}, I+\frac{1}{12} I I\right) \mathrm{d} x^{\prime}+\frac{1}{12} \int_{\Omega} Q_{2, A}\left(x^{\prime}, I I I\right) \mathrm{d} x^{\prime}+\frac{1}{180} \int_{\Omega} Q_{2, A}\left(x^{\prime}, I I\right) \mathrm{d} x^{\prime} \\
&= \mathcal{I}_{4}(V, \mathbb{S}),
\end{aligned}
$$

by a direct calculation. This completes the proof of Theorem 3.1 in view of (3.30).

\section{THE UPPeR BOUnd}

We now complete the proof of $\mathcal{I}_{4}$ being the $\Gamma$-limit of $h^{-4} E^{h}$, by proving that the lower bound (3.5) is optimal.

Theorem 4.1. Let $V \in W^{2,2}\left(\Omega, \mathbb{R}^{3}\right)$ and $\mathbb{S} \in L^{2}\left(\Omega, \mathbb{R}_{\text {sym }}^{2 \times 2}\right)$ satisfy:

$$
\begin{aligned}
& \operatorname{sym}\left(\left(\nabla y_{0}\right)^{t} \nabla V\right)=0, \\
& \mathbb{S} \in \mathcal{S}:=\operatorname{cl}_{L^{2}}\left\{\operatorname{sym}\left(\left(\nabla y_{0}\right)^{t} \nabla w\right) ; w \in W^{1,2}\left(\Omega, \mathbb{R}^{3}\right)\right\} .
\end{aligned}
$$


Then there exists a sequence $u^{h} \in W^{1,2}\left(\Omega^{h}, \mathbb{R}^{3}\right)$ such that assertions (i), (ii) and (iii) of Theorem 3.1 are satisfied with $R^{h}=\operatorname{Id}$ and $c^{h}=0$, and:

$$
\limsup _{h \rightarrow 0} \frac{1}{h^{4}} E^{h}\left(u^{h}\right) \leq \mathcal{I}_{4}(V, \mathbb{S}) .
$$

Proof. In the construction below, we will use the following notation. In view of (3.4), for every $F_{2 \times 2} \in \mathbb{R}^{2 \times 2}$ one can write:

$$
\mathcal{Q}_{2, A}\left(x^{\prime}, F_{2 \times 2}\right)=\min _{c \in \mathbb{R}^{3}}\left\{\mathcal{Q}_{3}\left(A^{-1}\left(F_{2 \times 2}^{*}+\operatorname{sym}\left(c \otimes e_{3}\right)\right) A^{-1}\right)\right\},
$$

where $F_{2 \times 2}^{*}$ denotes the $\mathbb{R}^{3 \times 3}$ matrix whose principal $2 \times 2$ minor equals $F_{2 \times 2}$. We will denote by $c\left(x^{\prime}, F_{2 \times 2}\right)$ the unique minimizer in $(4.3)$. Note that $c\left(x^{\prime}, \cdot\right)$ is a linear function of $F_{2 \times 2}$ and it depends only on its symmetric part $\left(\operatorname{sym} F_{2 \times 2}\right)$.

1. Since $\mathbb{S} \in \mathcal{S}$, there exists a sequence $w^{h} \in W^{1,2}\left(\Omega, \mathbb{R}^{3}\right)$ such that:

$$
\operatorname{sym}\left(\left(\nabla y_{0}\right)^{t} \nabla\left(w^{h}+\frac{1}{24} \vec{d}_{0}\right)\right) \rightarrow \mathbb{S} \quad \text { in } L^{2}\left(\Omega, \mathbb{R}^{2 \times 2}\right)
$$

and without loss of generality we can assume that each $w^{h}$ is smooth up to the boundary of $\Omega$, together with:

$$
\lim _{h \rightarrow 0} \sqrt{h}\left\|w^{h}\right\|_{W^{2, \infty}}=0 .
$$

Fix a small $\epsilon_{0} \in(0,1)$ and let $v^{h} \in W^{2, \infty}\left(\Omega, \mathbb{R}^{3}\right)$ be a sequence of Lipschitz deformations with the properties:

$$
\begin{gathered}
v^{h} \rightarrow V \quad \text { in } W^{2,2}\left(\Omega, \mathbb{R}^{3}\right), \\
h\left\|v^{h}\right\|_{W^{2, \infty}} \leq \epsilon_{0}, \\
\lim _{h \rightarrow 0} \frac{1}{h^{2}}\left|\left\{x^{\prime} \in \Omega ; v^{h}\left(x^{\prime}\right) \neq V\left(x^{\prime}\right)\right\}\right|=0 .
\end{gathered}
$$

We refer to [49] and [29] for the construction of such truncated sequence $v^{h}$. Define now $\vec{p}^{h} \in W^{1, \infty}\left(\Omega, \mathbb{R}^{3}\right)$ by:

$$
\vec{p}^{h}=\left(Q_{0}^{t}\right)^{-1}\left[\begin{array}{c}
-\left(\nabla v^{h}\right)^{t} \vec{b}_{0} \\
0
\end{array}\right]
$$

and also define the fields $\vec{q}^{h} \in W^{1, \infty}\left(\Omega, \mathbb{R}^{3}\right), \vec{k}_{0}$ smooth and $\tilde{r}^{h} \in L^{\infty}\left(\Omega, \mathbb{R}^{3}\right)$ such that:

$$
\begin{aligned}
Q_{0}^{t} \vec{q}^{h} & =\frac{1}{2} c\left(x^{\prime}, 2\left(\nabla y_{0}\right)^{t} \nabla w^{h}+\left(\nabla v^{h}\right)^{t} \nabla v^{h}\right)-\left[\begin{array}{c}
\left(\nabla w^{h}\right)^{t} \vec{b}_{0} \\
0
\end{array}\right]-\left[\begin{array}{c}
\left(\nabla v^{h}\right)^{t} \vec{p}^{h} \\
\frac{1}{2}\left|\vec{p}^{h}\right|^{2}
\end{array}\right], \\
Q_{0}^{t} \vec{k}_{0} & =c\left(x^{\prime},\left(\nabla y_{0}\right)^{t} \nabla \overrightarrow{d_{0}}+\left(\nabla \vec{b}_{0}\right)^{t} \nabla \vec{b}_{0}\right)-\left[\begin{array}{c}
\left(\nabla \vec{b}_{0}\right)^{t} \vec{d}_{0} \\
\left|\vec{d}_{0}\right|^{2}
\end{array}\right], \\
Q_{0}^{t} \tilde{r}^{h} & =c\left(x^{\prime},\left(\nabla y_{0}\right)^{t} \nabla \vec{p}^{h}+\left(\nabla v^{h}\right)^{t} \nabla \vec{b}_{0}\right)-\left[\begin{array}{c}
\left(\nabla v^{h}\right)^{t} \vec{d}_{0} \\
\left\langle\vec{p}^{h}, \vec{d}_{0}\right\rangle
\end{array}\right] .
\end{aligned}
$$


Finally, let $\vec{r}^{h} \in W^{1, \infty}\left(\Omega, \mathbb{R}^{3}\right)$ be such that:

$$
\lim _{h \rightarrow 0}\left\|\vec{r}^{h}-\tilde{r}^{h}\right\|_{L^{2}}=0, \quad \lim _{h \rightarrow 0} \sqrt{h}\left\|\vec{r}^{h}\right\|_{W^{1, \infty}}=0 .
$$

It follows from the definition of the minimizing map $c$, that:

$$
\begin{aligned}
& \mathcal{Q}_{3}\left(A^{-1}\left(2 Q_{0}^{t}\left[\nabla w^{h} \mid \vec{q}^{h}\right]+\left[\nabla v^{h} \mid \vec{p}^{h}\right]^{t}\left[\nabla v^{h} \mid \vec{p}^{h}\right]\right) A^{-1}\right) \\
& =\mathcal{Q}_{2, A}\left(x^{\prime}, 2\left(\nabla y_{0}\right)^{t} \nabla w^{h}+\left(\nabla v^{h}\right)^{t} \nabla v^{h}\right), \\
& \mathcal{Q}_{3}\left(A^{-1}\left(Q_{0}^{t}\left[\nabla \vec{d}_{0} \mid \vec{k}_{0}\right]+\left[\nabla \vec{b}_{0} \mid \vec{d}_{0}\right]^{t}\left[\nabla \vec{b}_{0} \mid \vec{d}_{0}\right]\right) A^{-1}\right) \\
& =\mathcal{Q}_{2, A}\left(x^{\prime},\left(\nabla y_{0}\right)^{t} \nabla \overrightarrow{d_{0}}+\left(\nabla \vec{b}_{0}\right)^{t} \nabla \vec{b}_{0}\right), \\
& \mathcal{Q}_{3}\left(A^{-1}\left(2 Q_{0}^{t}\left[\nabla \vec{p}^{h} \mid \tilde{r}^{h}\right]+2\left[\nabla v^{h} \mid \vec{p}^{h}\right]^{t}\left[\nabla \vec{b}_{0} \mid \vec{d}_{0}\right]^{t}\right) A^{-1}\right) \\
& =\mathcal{Q}_{2, A}\left(x^{\prime},\left(\nabla y_{0}\right)^{t} \nabla \vec{p}^{h}+\left(\nabla v^{h}\right)^{t} \nabla \vec{b}_{0}\right) .
\end{aligned}
$$

Moreover, we have the following pointwise bounds:

$$
\begin{aligned}
\left|\vec{p}^{h}\right| & \leq C\left|\nabla v^{h}\right| \\
\left|\nabla \vec{p}^{h}\right| & \leq C\left(\left|\nabla v^{h}\right|+\left|\nabla^{2} v^{h}\right|\right), \\
\left|\vec{q}^{h}\right| & \leq C\left(\left|\nabla w^{h}\right|+\left|\nabla v^{h}\right|^{2}+\left|\nabla v^{h}\right|\left|\vec{p}^{h}\right|+\left|\vec{p}^{h}\right|^{2}\right) \leq C\left(\left|\nabla w^{h}\right|+\left|\nabla v^{h}\right|^{2}\right), \\
\left|\nabla \vec{q}^{h}\right| & \leq C\left(\left|\nabla w^{h}\right|+\left|\nabla^{2} w^{h}\right|+\left|\nabla^{2} v^{h}\right|\left|\nabla v^{h}\right|+\left|\nabla v^{h}\right|^{2}\right) .
\end{aligned}
$$

2. Consider the sequence $u^{h} \in W^{1, \infty}\left(\Omega^{h}, \mathbb{R}^{3}\right)$ defined as:

$$
\begin{aligned}
u^{h}\left(x^{\prime}, x_{3}\right)= & y_{0}\left(x^{\prime}\right)+h v^{h}\left(x^{\prime}\right)+h^{2} w^{h}\left(x^{\prime}\right)+x_{3} \vec{b}_{0}\left(x^{\prime}\right)+\frac{x_{3}^{2}}{2} \vec{d}_{0}\left(x^{\prime}\right) \\
& +\frac{x_{3}^{3}}{6} \vec{k}_{0}\left(x^{\prime}\right)+h x_{3} \vec{p}^{h}\left(x^{\prime}\right)+h^{2} x_{3} \vec{q}^{h}\left(x^{\prime}\right)+\frac{h x_{3}^{2}}{2} \vec{r}^{h}\left(x^{\prime}\right) .
\end{aligned}
$$

For every $\left(x^{\prime}, x_{3}\right) \in \Omega^{1}$ we write:

$$
\nabla u^{h}\left(x^{\prime}, h x_{3}\right)=Q_{0}\left(x^{\prime}\right)+Z_{1}^{h}\left(x^{\prime}, x_{3}\right)+Z_{2}^{h}\left(x^{\prime}, x_{3}\right),
$$

where:

$$
\begin{aligned}
& Z_{1}^{h}\left(x^{\prime}, x_{3}\right)=h\left[\nabla v^{h} \mid \vec{p}^{h}\right]+h^{2}\left[\nabla w^{h} \mid \vec{q}^{h}\right]+h x_{3}\left[\nabla \vec{b}_{0} \mid \vec{d}_{0}\right]+\frac{h^{2} x_{3}^{2}}{2}\left[\nabla \vec{d}_{0} \mid \vec{k}_{0}\right]+h^{2} x_{3}\left[\nabla \vec{p}^{h} \mid \vec{r}^{h}\right], \\
& Z_{2}^{h}\left(x^{\prime}, x_{3}\right)=\frac{h^{3} x_{3}^{3}}{6}\left[\nabla \vec{k}_{0} \mid 0\right]+h^{3} x_{3}\left[\nabla \vec{q}^{h} \mid 0\right]+\frac{h^{3} x_{3}}{2}\left[\nabla \vec{r}^{h} \mid 0\right] .
\end{aligned}
$$

Since $Q_{0} A^{-1} \in S O(3)$, we get:

$$
\nabla u^{h} A^{-1}\left(x^{\prime}, h x_{3}\right)=Q_{0} A^{-1}\left(\operatorname{Id}_{3}+A^{-1} Q_{0}^{t} Z_{1}^{h} A^{-1}+A^{-1} Q_{0}^{t} Z_{2}^{h} A^{-1}\right)
$$


and, in view of (4.6), (4.8) and (4.10), there follows for $h$ sufficiently small:

$$
\begin{gathered}
\left\|A^{-1} Q_{0}^{t} Z_{1}^{h} A^{-1}+A^{-1} Q_{0}^{t} Z_{2}^{h} A^{-1}\right\|_{L^{\infty}} \\
\leq C\left(h\left\|\nabla v^{h}\right\|_{L^{\infty}}+h\left\|\vec{p}^{h}\right\|_{L^{\infty}}+h^{2}\left\|\nabla w^{h}\right\|_{L^{\infty}}+h^{2}\left\|\vec{q}^{h}\right\|_{L^{\infty}}+h\left\|\nabla \vec{b}_{0}\right\|_{L^{\infty}}+h\left\|\vec{d}_{0}\right\|_{L^{\infty}}\right. \\
\quad+h^{2}\left\|\nabla \overrightarrow{d_{0}}\right\|_{L^{\infty}}+h^{2}\left\|\vec{k}_{0}\right\|_{L^{\infty}}+h^{2}\left\|\nabla \vec{p}^{h}\right\|_{L^{\infty}}+h^{2}\left\|\vec{r}^{h}\right\|_{L^{\infty}}+h^{3}\left\|\nabla \vec{k}_{0}\right\|_{L^{\infty}} \\
\left.\quad+h^{3}\left\|\nabla \vec{q}^{h}\right\|_{L^{\infty}}+h^{3}\left\|\nabla \vec{r}^{h}\right\|_{L^{\infty}}\right) \leq C \epsilon_{0} .
\end{gathered}
$$

By the left polar decomposition, there exists a further rotation $R \in S O(3)$ such that:

$$
\begin{aligned}
R \nabla u^{h} A^{-1}= & \left(\left(\operatorname{Id}_{3}+A^{-1} Q_{0}^{t} Z_{1}^{h} A^{-1}+A^{-1} Q_{0}^{t} Z_{2}^{h} A^{-1}\right)^{t}\left(\operatorname{Id}_{3}+A^{-1} Q_{0}^{t} Z_{1}^{h} A^{-1}+A^{-1} Q_{0}^{t} Z_{2}^{h} A^{-1}\right)\right)^{1 / 2} \\
= & \left(\operatorname{Id}_{3}+2 A^{-1} \operatorname{sym}\left(Q_{0}^{t} Z_{1}^{h}\right) A^{-1}+A^{-1}\left(Z_{1}^{h}\right)^{t} Z_{1}^{h} A^{-1}+\mathcal{O}\left(\left|Z_{2}^{h}\right|\right)\right)^{1 / 2} \\
= & \operatorname{Id}_{3}+A^{-1} \operatorname{sym}\left(Q_{0}^{t} Z_{1}^{h}\right) A^{-1}+\frac{1}{2} A^{-1}\left(Z_{1}^{h}\right)^{t} Z_{1}^{h} A^{-1} \\
& +\mathcal{O}\left(\left|\operatorname{sym}\left(Q_{0}^{t} Z_{1}^{h}\right)+\left(Z_{1}^{h}\right)^{t} Z_{1}^{h}\right|^{2}\right)+\mathcal{O}\left(\left|Z_{2}^{h}\right|\right) .
\end{aligned}
$$

3. Consider the set:

$$
\Omega_{h}=\left\{\left(x^{\prime}, x_{3}\right) \in \Omega ; v^{h}\left(x^{\prime}\right)=V\left(x^{\prime}\right)\right\} .
$$

Note that on $\Omega_{h}$ we have: $\vec{p}^{h}=\vec{p}$ and $Q_{0}^{t}\left[\nabla v^{h} \mid \vec{p}^{h}\right] \in s o(3)$. Using Taylor's expansion, it follows that:

$$
\frac{1}{h^{4}} \int_{\Omega_{h}} W\left(\nabla u\left(x^{\prime}, h x_{3}\right) A^{-1}\right) \mathrm{d} x=\frac{1}{2 h^{4}} \int_{\Omega_{h}} \mathcal{Q}_{3}\left(A^{-1}\left(Q_{0}^{t} Z_{1}^{h}+\frac{1}{2}\left(Z_{1}^{h}\right)^{t} Z_{1}^{h}\right) A^{-1}\right) \mathrm{d} x+\mathcal{E}_{1}^{h},
$$

where the error term $\mathcal{E}_{1}^{h}$ can be estimated by:

$$
\left|\mathcal{E}_{1}^{h}\right| \leq \frac{C}{h^{4}} \int_{\Omega_{h}}\left|2 \operatorname{sym}\left(Q_{0}^{t} Z_{1}^{h}\right)+\left(Z_{1}^{h}\right)^{t} Z_{1}^{h}\right|^{3}+\left|Z_{2}^{h}\right|^{2}+\left|2 \operatorname{sym}\left(Q_{0}^{t} Z_{1}^{h}\right)+\left(Z_{1}^{h}\right)^{t} Z_{1}^{h}\right|\left|Z_{2}^{h}\right| \mathrm{d} x .
$$

Now on $\Omega_{h}$ we also have, by (4.10):

$$
\begin{aligned}
\left|2 \operatorname{sym}\left(Q_{0}^{t} Z_{1}^{h}\right)+\left(Z_{1}^{h}\right)^{t} Z_{1}^{h}\right| & \leq C\left(h^{2}\left|\nabla w^{h}\right|+h^{2}\left|\nabla v^{h}\right|^{2}+h^{2}+h^{2}\left|\nabla v^{h}\right|+h^{2}\left|\nabla^{2} v^{h}\right|+h^{2}\left|\vec{r}^{h}\right|\right), \\
\left|Z_{2}^{h}\right| & \leq C h^{3}\left(1+\left|\nabla \vec{q}^{h}\right|+\left|\nabla \vec{r}^{h}\right|\right) \\
& \leq C h^{3}\left(1+\left|\nabla w^{h}\right|+\left|\nabla^{2} w^{h}\right|+\left|\nabla^{2} v^{h}\right|\left|\nabla v^{h}\right|+\left|\nabla v^{h}\right|^{2}+\left|\nabla \vec{r}^{h}\right|\right),
\end{aligned}
$$


and therefore, in view of (4.5), (4.8), (4.6) and $V \in W^{2,2}$ :

$$
\begin{aligned}
\frac{1}{h^{4}} \int_{\Omega_{h}} & \left|2 \operatorname{sym}\left(Q_{0}^{t} Z_{1}^{h}\right)+\left(Z_{1}^{h}\right)^{t} Z_{1}^{h}\right|^{3} \mathrm{~d} x \\
\leq & \frac{C}{h^{4}} \int_{\Omega_{h}} h^{6}\left|\nabla w^{h}\right|^{3}+h^{6}\left|\nabla v^{h}\right|^{6}+h^{6}+h^{6}\left|\nabla v^{h}\right|^{3}+h^{6}\left|\nabla^{2} v^{h}\right|^{3}+h^{6}\left|\vec{r}^{h}\right|^{3} \mathrm{~d} x \\
\leq & \frac{C}{h^{4}}\left(h^{2}\left\|\nabla w^{h}\right\|_{L^{\infty}}\left(h^{2}\left\|\nabla w^{h}\right\|_{L^{2}}\right)^{2}+h^{6}\|\nabla V\|_{L^{6}}^{6}+h^{6}|\Omega|+h^{6}\|\nabla V\|_{L^{3}}^{3}\right. \\
& \left.\quad+h^{6}\left\|\nabla^{2} v^{h}\right\|_{L^{\infty}}\left\|\nabla^{2} V\right\|_{L^{2}}^{2}+\left(\sqrt{h}\left\|\vec{r}^{h}\right\|_{L^{\infty}}\right)^{3} h^{9 / 2}\right) \rightarrow 0 \quad \text { as } h \rightarrow 0 .
\end{aligned}
$$

Analogously:

$$
\begin{gathered}
\frac{1}{h^{4}} \int_{\Omega_{h}}\left|Z_{2}^{h}\right|^{2} \mathrm{~d} x \leq \frac{C}{h^{4}} \int_{\Omega_{h}} h^{5}+\left(h\left\|\nabla v^{h}\right\|_{L^{\infty}}\right)^{2} h^{4}\left|\nabla^{2} v^{h}\right|^{2}+h^{6}\left|\nabla v^{h}\right|^{4} \mathrm{~d} x \rightarrow 0 \quad \text { as } h \rightarrow 0, \\
\frac{1}{h^{4}} \int_{\Omega_{h}}\left|2 \operatorname{sym}\left(Q_{0}^{t}\right) Z_{1}^{h}+\left(Z_{1}^{h}\right)^{t} Z_{1}^{h}\right|\left|Z_{2}^{h}\right| \mathrm{d} x \\
\leq \frac{C}{h^{4}} \int_{\Omega_{h}}\left(h^{5}\left|\nabla w^{h}\right|^{2}+h^{5}\left|\nabla^{2} w^{h}\right|^{2}+h^{5}\left|\nabla v^{h}\right|^{2}+h^{5}+h^{5}|\nabla V|+h^{5}\left|\nabla^{2} V\right|+h^{5}\left|\vec{r}^{h}\right|\right. \\
\left.+h^{5}|\nabla V|^{2}\left|\nabla^{2} V\right|+h^{5}|\nabla V|\left|\nabla^{2} V\right|^{2}\right) \mathrm{d} x \leq C \epsilon_{0} .
\end{gathered}
$$

We therefore conclude that:

$$
\limsup _{h \rightarrow 0}\left|\mathcal{E}_{1}^{h}\right| \leq C \epsilon_{0}
$$

4. Consider now the error due to integrating on the residual subdomain:

$\mathcal{E}_{2}^{h}=\frac{1}{h^{4}} \int_{\Omega^{1} \backslash \Omega_{h}} W\left(\nabla u^{h} A^{-1}\left(x^{\prime}, h x_{3}\right)\right) \mathrm{d} x \leq \frac{C}{h^{4}} \int_{\Omega^{1} \backslash \Omega_{h}}\left|2 \operatorname{sym}\left(Q_{0}^{t} Z_{1}^{h}\right)+\left(Z_{1}^{h}\right)^{t} Z_{1}^{h}\right|^{2}+\left|Z_{2}^{h}\right|^{2} \mathrm{~d} x$.

Observe that, since the matrix field $\left[\nabla v^{h} \mid \vec{p}^{h}\right]$ is Lipschitz, we have:

$$
\begin{aligned}
\left|\operatorname{sym}\left(Q_{0}^{t}\left[\nabla v^{h} \mid \vec{p}^{h}\right]\right)\left(x^{\prime}\right)\right| & \leq C\left\|\nabla v^{h}\right\|_{W^{1, \infty}} \operatorname{dist}\left(x^{\prime},\left\{v^{h}=V\right\}\right) \\
& \leq \frac{C \epsilon_{0}}{h} \operatorname{dist}\left(x^{\prime},\left\{v^{h}=V\right\}\right) \rightarrow 0 \quad \text { in } L^{\infty}(\Omega) .
\end{aligned}
$$

The last inequality above follows by a standard argument by contradiction. If there was a sequence $x^{h} \in \Omega$ such that $\operatorname{dist}\left(x^{h},\left\{v^{h}=V\right\}\right) \geq c h$, this would imply that: $\mid\left\{x^{\prime} ; v^{h}\left(x^{\prime}\right) \neq\right.$ 
$\left.V\left(x^{\prime}\right)\right\}|\geq| \Omega \cap B\left(x^{h}, c h\right) \mid \geq c h^{2}$, contradicting (4.6). Consequently, by (4.5), (4.8), (4.6):

$$
\begin{aligned}
\left|\mathcal{E}_{2}^{h}\right| \leq & \frac{C}{h^{4}} \int_{\Omega^{1} \backslash \Omega_{h}} h^{2}\left|\operatorname{sym}\left(Q_{0}^{t}\left[\nabla v^{h} \mid \vec{p}^{h}\right]\right)\right| \mathrm{d} x \\
& \quad+\frac{C}{h^{4}} \int_{\Omega^{1} \backslash \Omega_{h}} h^{4}\left|\nabla w^{h}\right|^{2}+h^{4}\left|\nabla v^{h}\right|^{4}+h^{4}\left|\nabla^{2} v^{h}\right|^{2}+h^{4}\left|\vec{r}^{h}\right|^{2}+h^{4}+h^{6}\left|\nabla v^{h}\right|^{4} \mathrm{~d} x \\
\leq & \frac{C}{h^{4}} o\left(h^{2}\right)\left|\Omega^{1} \backslash \Omega_{h}\right|+\frac{C}{h^{4}} \sqrt{h}\left\|\nabla w^{h}\right\|_{L^{\infty}} h^{7 / 2}\left|\Omega^{1} \backslash \mathcal{U}^{h}\right|^{1 / 2}\left\|\nabla w^{h}\right\|_{L^{2}} \\
& +C\left|\Omega^{1} \backslash \mathcal{U}^{h}\right|\left\|\nabla v^{h}\right\|_{L^{8}}^{4}+C h\left\|\nabla^{2} v^{h}\right\|_{L^{\infty}} \frac{1}{h}\left\|\nabla^{2} v^{h}\right\|_{L^{2}}\left|\Omega^{1} \backslash \mathcal{U}^{h}\right|^{1 / 2}+\frac{1}{h}\left(\sqrt{h}\left\|\vec{r}^{h}\right\|_{L^{\infty}}\right)^{2}\left|\Omega^{1} \backslash \mathcal{U}^{h}\right| \\
& +\left(h\left\|\nabla^{2} v^{h}\right\|_{L^{\infty}}\right)^{2}\left\|\nabla v^{h}\right\|_{L^{4}}^{2}\left|\Omega^{1} \backslash \mathcal{U}^{h}\right|^{1 / 2} \quad \rightarrow 0 \quad \text { as } h \rightarrow 0 .
\end{aligned}
$$

Thus:

$$
\limsup _{h \rightarrow 0} \frac{1}{h^{4}} E^{h}\left(u^{h}\right) \leq \limsup _{h \rightarrow 0} \frac{1}{h^{4}} \int_{\Omega_{h}} \frac{1}{2} \mathcal{Q}_{3}\left(A^{-1}\left(\operatorname{sym}\left(Q_{0}^{t} Z_{1}^{h}\right)+\frac{1}{2}\left(Z_{1}^{h}\right)^{t} Z_{1}^{h}\right) A^{-1}\right) \mathrm{d} x+C \epsilon_{0} .
$$

Now on $\Omega_{h}$ we have:

$$
\begin{aligned}
2 \operatorname{sym}( & \left.Q_{0}^{t} Z_{1}^{h}\right)+\left(Z_{1}^{h}\right)^{t} Z_{1}^{h} \\
= & 2 h^{2}\left(\operatorname{sym}\left(Q_{0}^{t}\left[\nabla w^{h} \mid \vec{q}^{h}\right]\right)+\frac{x_{3}^{2}}{2} \operatorname{sym}\left(Q_{0}^{t}\left[\nabla \vec{d}_{0} \mid \vec{k}_{0}\right]\right)+x_{3} \operatorname{sym}\left(Q_{0}^{t}\left[\nabla \vec{p} \mid \vec{r}^{h}\right]\right)\right) \\
& +h^{2}\left([\nabla V \mid \vec{p}]^{t}[\nabla V \mid \vec{p}]+x_{3}^{2}\left[\nabla \vec{b}_{0} \mid \vec{d}_{0}\right]^{t}\left[\nabla \vec{b}_{0} \mid \vec{d}_{0}\right]+2 x_{3} \operatorname{sym}\left([\nabla V \mid \vec{p}]^{t}\left[\nabla \vec{b}_{0} \mid \vec{d}_{0}\right]\right)\right)+\mathcal{E}^{h},
\end{aligned}
$$

where the present error $\mathcal{E}^{h}$ is estimated by:

$$
\begin{aligned}
\left|\mathcal{E}^{h}\right| \leq C\left(h^{3}|\nabla V|\left|\nabla w^{h}\right|+h^{3}|\nabla V|+h^{3}|\nabla V||\nabla \vec{p}|+h^{3}|\nabla V|\left|\vec{r}^{h}\right|\right. \\
+h^{4}\left|\nabla w^{h}\right|^{2}+h^{3}\left|\nabla w^{h}\right|+h^{4}\left|\nabla w^{h}\right|\left|\nabla \vec{p}+h^{4}\right| \nabla w^{h}|| \vec{r}^{h} \mid+h^{3} \\
\left.\quad+h^{3}|\nabla \vec{p}|+h^{3}\left|\vec{r}^{h}\right|+h^{4}+h^{4}|\nabla \vec{p}|+h^{4}\left|\vec{r}^{h}\right|+h^{4}|\nabla \vec{p}|^{2}+h^{4}\left|\vec{r}^{h}\right|^{2}\right) \\
\leq C h^{2}\left(o(1) \sqrt{h}|\nabla V|+\epsilon_{0}^{2}\left|\nabla^{2} V\right|+o(1) \sqrt{h}+o(1) \epsilon_{0} \sqrt{h}\right) .
\end{aligned}
$$


Consequently:

$$
\begin{aligned}
& \limsup _{h \rightarrow 0} \frac{1}{h^{4}} E^{h}\left(u^{h}\right) \\
& \leq \limsup _{h \rightarrow 0} \frac{1}{2} \int_{\Omega_{h}} \mathcal{Q}_{3}\left(A ^ { - 1 } \left(\operatorname{sym}\left(Q_{0}^{t}\left[\nabla w^{h} \mid \vec{q}^{h}\right]\right)+\frac{1}{2} x_{3}^{2} \operatorname{sym}\left(Q_{0}^{t}\left[\nabla \vec{d}_{0} \mid \vec{k}_{0}\right]\right)\right.\right. \\
& \quad+x_{3} \operatorname{sym}\left(Q_{0}^{t}\left[\nabla \vec{p} \mid \vec{r}^{h}\right]\right)+\frac{1}{2}[\nabla V \mid \vec{p}]^{t}[\nabla V \mid \vec{p}] \\
&\left.\left.+\frac{1}{2} x_{3}^{2}\left[\nabla \vec{b}_{0} \mid \vec{d}_{0}\right]^{t}\left[\nabla \vec{b}_{0} \mid \vec{d}_{0}\right]+x_{3} \operatorname{sym}\left([\nabla V \mid \vec{p}]^{t}\left[\nabla \vec{b}_{0} \mid \vec{d}_{0}\right]\right)\right) A^{-1}\right) \mathrm{d} x+C \epsilon_{0} \\
& \quad \limsup _{h \rightarrow 0} \frac{1}{2} \int_{\Omega_{h}} \mathcal{Q}_{3}\left(A ^ { - 1 } \left(\operatorname{sym}\left(Q_{0}^{t}\left[\nabla w^{h} \mid \vec{q}^{h}\right]\right)+\frac{1}{2}[\nabla V \mid \vec{p}]^{t}[\nabla V \mid \vec{p}]\right.\right. \\
&\left.\left.\quad+\frac{1}{2} x_{3}^{2} \operatorname{sym}\left(Q_{0}^{t}\left[\nabla \vec{d}_{0} \mid \vec{k}_{0}\right]\right)+\frac{1}{2} x_{3}^{2}\left[\nabla \vec{b}_{0} \mid \vec{d}_{0}\right]^{t}\left[\nabla \vec{b}_{0} \mid \vec{d}_{0}\right]\right) A^{-1}\right) \\
&+\mathcal{Q}_{3}\left(A^{-1}\left(x_{3} \operatorname{sym}\left(Q_{0}^{t}\left[\nabla \vec{p} \mid \vec{r}^{h}\right]\right)+x_{3} \operatorname{sym}\left([\nabla V \mid \vec{p}]^{t}\left[\nabla \vec{b}_{0} \mid \vec{d}_{0}\right]\right)\right) A^{-1}\right) \mathrm{d} x+C \epsilon_{0} .
\end{aligned}
$$

Denoting by:

$$
I_{1}\left(x^{\prime}\right)=\operatorname{sym}\left(\left(\nabla y_{0}\right)^{t} \nabla w^{h}\right)+\frac{1}{2}\left(\nabla v^{h}\right)^{t} \nabla v^{h}, \quad I_{2}\left(x^{\prime}\right)=\frac{1}{2} \operatorname{sym}\left(\left(\nabla y_{0}\right)^{t} \nabla \overrightarrow{d_{0}}\right)+\left(\nabla \vec{b}_{0}\right)^{t} \nabla \vec{b}_{0},
$$

we have:

$$
\begin{aligned}
\mathcal{Q}_{3}( & \left.A^{-1}\left(I_{1}^{*}\left(x^{\prime}\right)+\operatorname{sym}\left(c\left(x^{\prime}, I_{1}\left(x^{\prime}\right)\right) \otimes e_{3}\right)+x_{3}^{2} I_{2}^{*}\left(x^{\prime}\right)+x_{3}^{2} \operatorname{sym}\left(c\left(x^{\prime}, I_{2}\left(x^{\prime}\right)\right) \otimes e_{3}\right)\right) A^{-1}\right) \\
& =\mathcal{Q}_{3}\left(A^{-1}\left(\left(I_{1}\left(x^{\prime}\right)+x_{3}^{2} I_{2}\left(x^{\prime}\right)\right)^{*}+\operatorname{sym}\left(c\left(x^{\prime}, I_{1}\left(x^{\prime}\right)+x_{3}^{2} I_{2}\left(x^{\prime}\right)\right) \otimes e_{3}\right)\right) A^{-1}\right) \\
& =\mathcal{Q}_{2, A}\left(\left(I_{1}\left(x^{\prime}\right)+x_{3}^{2} I_{2}\left(x^{\prime}\right)\right),\right.
\end{aligned}
$$

where we have used the definition and linearity of the minimizing map $c$. Recalling the definitions of the curvature forms $I\left(x^{\prime}\right), I I\left(x^{\prime}\right)$ and $I I I\left(x^{\prime}\right)$ in $(3.31)$, observe that $I_{2}\left(x^{\prime}\right)=$ $2 I I\left(x^{\prime}\right)$ and that $\frac{1}{2} I_{1}$ converges to $I$ in $L^{2}$ by (4.4). Hence:

$$
\begin{aligned}
\limsup _{h \rightarrow 0} \frac{1}{h^{4}} E^{h}\left(u^{h}\right) & \leq \frac{1}{2} \int_{\Omega^{1}} \mathcal{Q}_{2, A}\left(I\left(x^{\prime}\right)+x_{3}^{2} I I\left(x^{\prime}\right)\right) \mathrm{d} x+\frac{1}{2} \int_{\Omega^{1}} \mathcal{Q}_{2, A}\left(x_{3} I I I\left(x^{\prime}\right)\right) \mathrm{d} x+C \epsilon_{0} \\
& =\mathcal{I}_{4}(V, \mathbb{S})+C \epsilon_{0} .
\end{aligned}
$$

Since $\epsilon_{0}>0$ was arbitrary, the proof is achieved by a diagonal argument.

\section{Discussion of the von Kármán-Like functional (3.5)}

Theorems 3.1 and 4.1 imply, as usual in the setting of $\Gamma$-convergence, convergence of almost-minimizers:

Corollary 5.1. If $u^{h} \in W^{1,2}\left(\Omega^{h}, \mathbb{R}^{3}\right)$ is a minimizing sequence to $h^{-4} E^{h}$, that is:

$$
\lim _{h \rightarrow 0}\left(\frac{1}{h^{4}} E^{h}\left(u^{h}\right)-\inf \frac{1}{h^{4}} E^{h}\right)=0,
$$


then the appropriate renormalizations $y^{h}=\left(\bar{R}^{h}\right)^{t} u^{h}\left(x^{\prime}, h x_{3}\right)-c^{h} \in W^{1,2}\left(\Omega^{1}, \mathbb{R}^{3}\right)$ obey the convergence statements of Theorem 3.1 (i), (ii), (iii). The convergence of $h^{-1} \operatorname{sym}\left(\left(\nabla y_{0}\right)^{t} \nabla V^{h}\right)$ to $\mathbb{S}$ in (iii) is strong in $L^{2}(\Omega)$. Moreover, any limit $(V, \mathbb{S})$ minimizes the functional $\mathcal{I}_{4}$.

Proof. The proof is standard. The only possibly nontrivial part is the strong convergence of the scaled tangential strains in (iii), which can be deduced as in Theorem 2.5 in [41].

Let us now compare the functional (3.5) with the von-Kármán theory of thin shells that has been derived in [41]. Recall that when $S$ is a smooth $2 \mathrm{~d}$ surface in $\mathbb{R}^{3}$, the $\Gamma$-limit of the scaled elastic energies $h^{-4}\left(\frac{1}{h} \int_{S^{h}} W\left(\nabla u^{h}\right)\right)$ on thin shells $S^{h}$ with mid-surface $S$, is:

$$
\tilde{\mathcal{I}}_{4, S}(\tilde{V}, \tilde{\mathbb{S}})=\frac{1}{2} \int_{S} \mathcal{Q}_{2}\left(\tilde{\mathbb{S}}-\frac{1}{2}\left(\tilde{A}^{2}\right)_{\tan }\right) \mathrm{d} y+\frac{1}{24} \int_{S} \mathcal{Q}_{2}\left((\nabla(\tilde{A} \vec{N})-\tilde{A} \Pi)_{t a n}\right) \mathrm{d} y
$$

Above, $\Pi$ stands for the shape operator of $S$ and $\vec{N}$ is the unit normal vector to $S$. The subscript tan means taking the restriction of a quadratic form (or an operator) to the tangent space $T_{y} S$. The arguments of $\tilde{\mathcal{I}}_{4, S}$ are:

(i) First order infinitesimal isometries $\tilde{V}$ on $S$. These are vector fields $\tilde{V} \in W^{2,2}\left(S, \mathbb{R}^{3}\right)$ with skew symmetric covariant derivative, so that one may define:

$$
\tilde{A} \in W^{1,2}(S, s o(3)) \quad \text { with } \quad \tilde{A}(y) \tau=\partial_{\tau} \tilde{V}(y) \quad \forall y \in S \quad \forall \tau \in T_{y} S
$$

(ii) Finite strains $\tilde{\mathbb{S}}$ on $S$. These are tensor fields $\tilde{\mathbb{S}} \in L^{2}\left(S, \mathbb{R}_{\text {sym }}^{2 \times 2}\right)$ such that:

$$
\tilde{\mathbb{S}}=L^{2}-\lim _{h \rightarrow 0} \operatorname{sym}\left(\nabla \tilde{w}_{h}\right)_{t a n} \quad \text { for some } \quad \tilde{w}_{h} \in W^{1,2}\left(S, \mathbb{R}^{3}\right) .
$$

In the present setting, denote $S=y_{0}(\Omega)$ and observe that the 1-1 correspondence between $\tilde{V}$ in (5.2) and $V$ in (3.3) is given by the change of variables $V=\tilde{V} \circ y_{0}$. The skew-symmetric tensor field $\tilde{A}$ on $T_{y} S$ is then uniquely given by:

$$
\tilde{A}\left(y_{0}\left(x^{\prime}\right)\right) \partial_{e} y_{0}=\partial_{e} V\left(x^{\prime}\right) \text { and } \tilde{A} \vec{b}_{0}=\vec{p} \quad \forall e \in \mathbb{R}^{2},
$$

and the finite strains in (5.3) are related to (4.1) by:

$$
\left\langle\tilde{\mathbb{S}}\left(y_{0}\left(x^{\prime}\right)\right) \partial_{e} y_{0}, \partial_{e} y_{0}\right\rangle=\left\langle\mathbb{S}\left(x^{\prime}\right) e, e\right\rangle \quad \forall e \in \mathbb{R}^{2}
$$

Recall that the first of the two terms in the functional (5.1) measures the difference of order $h^{2}$, between the (Euclidean) metric on $S$ and the metric of the deformed surface. Indeed, the amount of stretching of $S$ in the direction $\tau \in T_{y} S$, induced by the deformation $u_{h}=i d+h \tilde{V}+h^{2} \tilde{w}$, has the expansion:

$\left|\partial_{\tau} u_{h}\right|^{2}-|\tau|^{2}=h^{2}\left(2\left\langle\partial_{\tau} \tilde{w}, \tau\right\rangle+\left|\partial_{\tau} \tilde{V}\right|^{2}\right)+\mathcal{O}\left(h^{3}\right)=2 h^{2}\left(\langle(\operatorname{sym} \nabla \tilde{w}) \tau, \tau\rangle-\frac{1}{2}\left\langle\tilde{A}^{2} \tau, \tau\right\rangle\right)+\mathcal{O}\left(h^{3}\right)$.

The leading order quantity in the right hand side above coincides with:

$$
\langle(\operatorname{sym} \nabla w) e, e\rangle+\frac{1}{2}\left\langle\partial_{e} V, \partial_{e} V\right\rangle=\left\langle\left(\operatorname{sym} \nabla w+\frac{1}{2}(\nabla V)^{t} \nabla V\right) e, e\right\rangle,
$$

where we write $\tau=\partial_{e} y_{0}$, for any $e \in \mathbb{R}^{2}$. This is precisely the argument of the first term in $\mathcal{I}_{4}(V, \mathbb{S})$, modulo the correction $\left(\nabla \vec{b}_{0}\right)^{t} \nabla \vec{b}_{0}$ (equal to the third fundamental form on $S$ in case 
$\vec{b}_{0}=\vec{N}$ ), due to the incompatibility of the ambient Euclidean metric of $S^{h}$ with the given prestrain $G$ on $\Omega^{h}$.

The second term in (5.1) measures the difference of order $h$, between the shape operator $\Pi$ on $S$ and the shape operator $\Pi^{h}$ on the deformed surface $(i d+h \tilde{V})(S)$ whose unit normal we denote by $\vec{N}^{h}$. The amount of bending of $S$, in the direction $\tau \in T_{y} S$, induced by the deformation $u_{h}=i d+h \tilde{V}$ can be estimated by [41]:

$$
\begin{aligned}
(\operatorname{Id}+h \tilde{A})^{-1} \Pi^{h} & (\operatorname{Id}+h \tilde{A}) \tau-\Pi \tau=(\operatorname{Id}+h \tilde{A})^{-1}\left(\partial_{\tau} \vec{N}^{h}+\mathcal{O}\left(h^{2}\right)\right) \tau-\Pi \tau \\
& =(\operatorname{Id}+h \tilde{A})^{-1}\left((\operatorname{Id}+h \tilde{A}) \Pi \tau+h\left(\partial_{\tau} A\right) \vec{N}+\mathcal{O}\left(h^{2}\right)\right)-\Pi \tau \\
& =(\operatorname{Id}-h \tilde{A}) h\left(\partial_{\tau} \tilde{A}\right) \vec{N}+\mathcal{O}\left(h^{2}\right) \\
& =h\left(\partial_{\tau} \tilde{A}\right) \vec{N}+\mathcal{O}\left(h^{2}\right)=h(\nabla(\tilde{A} \vec{N})-\tilde{A} \Pi)+\mathcal{O}\left(h^{2}\right) .
\end{aligned}
$$

The leading order term in this expansion coincides with the term $\left(\nabla y_{0}\right)^{t} \nabla \vec{p}+(\nabla V)^{t} \nabla \vec{b}_{0}$ when $\vec{b}_{0}=\vec{N}$, because in view of $(5.4)$ :

$$
\begin{aligned}
\left\langle\left(\partial_{\tau} \tilde{A}\right) \vec{b}_{0}, \tau\right\rangle & =\left\langle\left(\partial_{e}\left(\tilde{A} \vec{b}_{0}\right), \partial_{e} y_{0}\right\rangle-\left\langle\left(\tilde{A} \partial_{e} \vec{b}_{0}, \partial_{e} y_{0}\right\rangle=\left\langle\left(\partial_{e} \vec{p}, \partial_{e} y_{0}\right\rangle+\left\langle\left(\partial_{e} \vec{b}_{0}, \tilde{A} \partial_{e} y_{0}\right\rangle\right.\right.\right.\right. \\
& =\left\langle\left(\nabla y_{0}\right)^{t} \nabla \vec{p} e, e\right\rangle-\left\langle(\nabla V)^{t} \nabla \vec{b}_{0} e, e\right\rangle
\end{aligned}
$$

where we again wrote $\tau=\partial_{e} y_{0} \in T_{y_{0}\left(x^{\prime}\right)} S$, for any $e \in \mathbb{R}^{2}$. This is precisely the argument in the second term in $\mathcal{I}_{4}(V, \mathbb{S})$.

In the next section we identify the geometric significance of the last term in (3.5).

\section{The scaling optimality}

In this section, we prove the following crucial result:

Theorem 6.1. Assume (1.5), together with:

$$
\operatorname{sym}\left(\left(\nabla y_{0}\right)^{t} \nabla \vec{d}_{0}\right)+\left(\nabla \vec{b}_{0}\right)^{t} \nabla \vec{b}_{0}=0,
$$

where $y_{0}, \vec{b}_{0}$ and $\vec{d}_{0}$ are defined in (1.7), (1.8), (1.10). Then the metric $G$ is flat, i.e. $\operatorname{Riem}(G) \equiv 0$ in $\Omega^{h}$. Equivalently: $\min E^{h}=0$ for all $h$.

Observe that when $\vec{b}_{0}=\vec{N}$, then by (1.10) there must be $\vec{d}_{0}=0$, and hence condition (6.1) becomes: $\vec{N} \equiv$ const. This is consistent with our previous observation that when $G e_{3}=e_{3}$, then already condition (1.7) is enough to conclude immersability of $G$ in $\mathbb{R}^{3}$. Equivalently, $G_{2 \times 2}$ is immersible in $\mathbb{R}^{2}$, so that indeed $y_{0}(\Omega)$ must be planar in this case.

Towards a proof of Theorem 6.1, recall that Riem $(G)$ is the covariant Riemann curvature tensor, whose components $R_{\ldots .}$ and their relation to the contravariant curvatures in $R_{\ldots}$ are:

$$
\begin{aligned}
R_{i k l m} & =\frac{1}{2}\left(\partial_{k l} G_{i m}+\partial_{i m} G_{k l}-\partial_{k m} G_{i l}-\partial_{i l} G_{k m}\right)+G_{n p}\left(\Gamma_{k l}^{n} \Gamma_{i m}^{p}-\Gamma_{k m}^{n} \Gamma_{i l}^{p}\right) \\
R_{i k l m} & =G_{i s} R_{k l m}^{s}
\end{aligned}
$$


where we used the Einstein summation convention and the Christoffel symbols:

$$
\Gamma_{k l}^{n}=\frac{1}{2} G^{n s}\left(\partial_{k} G_{s l}+\partial_{l} G_{s k}-\partial_{s} G_{k l}\right) .
$$

In view of the symmetries in $\operatorname{Riem}(G)$ of a 3 -dimensional metric $G$, its flatness is equivalent to the vanishing of the following curvatures:

$$
R_{1212}, R_{1213}, R_{1223}, R_{1313}, R_{1323}, R_{2323} .
$$

The proof of Theorem 6.1 is a consequence of the following observation.

Theorem 6.2. Assume (1.5) and let $y_{0}, \vec{b}_{0}$ and $\vec{d}_{0}$ be defined as in (1.7), (1.10). Then:

$$
\operatorname{sym}\left(\left(\nabla y_{0}\right)^{t} \nabla \overrightarrow{d_{0}}\right)+\left(\nabla \vec{b}_{0}\right)^{t} \nabla \vec{b}_{0}=\left[\begin{array}{ll}
R_{1313} & R_{1323} \\
R_{1323} & R_{2323}
\end{array}\right] .
$$

Proof. 1. We have:

$$
\begin{aligned}
& R_{1313}=-\frac{1}{2} \partial_{11} G_{33}+G_{n p}\left(\Gamma_{13}^{n} \Gamma_{13}^{p}-\Gamma_{11}^{n} \Gamma_{33}^{p}\right), \\
& R_{2323}=-\frac{1}{2} \partial_{22} G_{33}+G_{n p}\left(\Gamma_{23}^{n} \Gamma_{23}^{p}-\Gamma_{22}^{n} \Gamma_{33}^{p}\right), \\
& R_{1323}=-\frac{1}{2} \partial_{12} G_{33}+G_{n p}\left(\Gamma_{13}^{n} \Gamma_{23}^{p}-\Gamma_{12}^{n} \Gamma_{33}^{p}\right) .
\end{aligned}
$$

On the other hand, in view of (1.10):

$$
\begin{aligned}
\forall i, j=1,2 \quad \frac{1}{2}\left(\left\langle\partial_{i} y_{0}, \partial_{j} \vec{d}_{0}\right\rangle+\left\langle\partial_{j} y_{0}, \partial_{i} \vec{d}_{0}\right\rangle\right) & =\frac{1}{2}\left(\partial_{j}\left\langle\partial_{i} y_{0}, \vec{d}_{0}\right\rangle+\partial_{i}\left\langle\partial_{j} y_{0}, \overrightarrow{d_{0}}\right\rangle\right)-\left\langle\partial_{i j} y_{0}, \vec{d}_{0}\right\rangle \\
& =-\frac{1}{2} \partial_{i j} G_{33}-\left\langle\partial_{i j} y_{0}, \vec{d}_{0}\right\rangle
\end{aligned}
$$

because: $\partial_{j}\left\langle\partial_{i} y_{0}, \vec{d}_{0}\right\rangle+\partial_{i}\left\langle\partial_{j} y_{0}, \vec{d}_{0}\right\rangle=-\partial_{i j}\left|\vec{b}_{0}\right|^{2}=-\partial_{i j} G_{33}$. Consequently, the formula (6.3) will follow, if we establish:

$$
\forall i, j=1,2 \quad\left\langle\partial_{i j} y_{0}, \vec{d}_{0}\right\rangle=G_{n p} \Gamma_{i j}^{n} \Gamma_{33}^{p} \quad \text { and } \quad\left\langle\partial_{i} \vec{b}_{0}, \partial_{j} \vec{b}_{0}\right\rangle=G_{n p} \Gamma_{i 3}^{n} \Gamma_{j 3}^{p} .
$$

2. Before proving (6.4) we gather some useful formulas. Note that $\partial_{i} G=2 \operatorname{sym}\left(\left(\partial_{i} Q\right)^{t} Q\right)$ for $i=1,2$. Therefore, by direct inspection:

$$
\forall i, j, k=1,2 \quad\left\langle\partial_{i j} y_{0}, \partial_{k} y_{0}\right\rangle=\frac{1}{2}\left(\partial_{i} G_{k j}+\partial_{j} G_{k i}-\partial_{k} G_{i j}\right) .
$$

Also, recall that condition (1.7) is equivalent to (see [6], proof of Theorem 5.3, formula (5.8)):

$$
\forall i, j=1,2 \quad\left\langle\partial_{i j} y_{0}, \vec{b}_{0}\right\rangle=\frac{1}{2}\left(\partial_{i} G_{j 3}+\partial_{j} G_{i 3}\right) .
$$

Therefore, for all $i, j=1,2$ :

$$
\begin{aligned}
& \left\langle\partial_{j} y_{0}, \partial_{i} \vec{b}_{0}\right\rangle=\partial_{i}\left\langle\partial_{j} y_{0}, \vec{b}_{0}\right\rangle-\left\langle\partial_{i j} y_{0}, \vec{b}_{0}\right\rangle=\frac{1}{2}\left(\partial_{i} G_{j 3}-\partial_{j} G_{i 3}\right), \\
& \left\langle\partial_{i} \vec{b}_{0}, \vec{b}_{0}\right\rangle=\frac{1}{2} \partial_{i} G_{33} .
\end{aligned}
$$


We now express $\partial_{i j} y_{0}, \partial_{i} \vec{b}_{0}$ and $\vec{d}_{0}$ in the basis $\left\{\partial_{1} y_{0}, \partial_{2} y_{0}, \vec{b}_{0}\right\}$, writing:

$$
\begin{aligned}
& \partial_{i j} y_{0}=\alpha_{i j}^{1} \partial_{1} y_{0}+\alpha_{i j}^{2} \partial_{2} y_{0}+\alpha_{i j}^{3} \vec{b}_{0}, \\
& \partial_{i} \vec{b}_{0}=\beta_{i}^{1} \partial_{1} y_{0}+\beta_{i}^{2} \partial_{2} y_{0}+\beta_{i}^{3} \vec{b}_{0}, \\
& \vec{d}_{0}=\gamma^{1} \partial_{1} y_{0}+\gamma^{2} \partial_{2} y_{0}+\gamma^{3} \vec{b}_{0} .
\end{aligned}
$$

By (6.5), (6.6), (6.7) and (1.10), it follows that:

$$
\begin{aligned}
G\left(\alpha_{i j}^{1}, \alpha_{i j}^{2}, \alpha_{i j}^{3}\right)^{t} & =G Q_{0}^{-1} \partial_{i j} y_{0}=Q_{0}^{t} \partial_{i j} y_{0} \\
& =\frac{1}{2}\left(\partial_{i} G_{1 j}+\partial_{j} G_{1 i}-\partial_{1} G_{i j}, \partial_{i} G_{2 j}+\partial_{j} G_{2 i}-\partial_{2} G_{i j}, \partial_{i} G_{3 j}+\partial_{j} G_{3 i}\right), \\
G\left(\beta_{i}^{1}, \beta_{i}^{2}, \beta_{i}^{3}\right)^{t}= & G Q_{0}^{-1} \partial_{i} \vec{b}_{0}=Q_{0}^{t} \partial \vec{b}_{0}=Q_{0}^{t} \partial_{i} \vec{b}_{0} \\
= & \frac{1}{2}\left(\partial_{i} G_{13}-\partial_{1} G_{i 3}, \partial_{i} G_{23}-\partial_{2} G_{i 3}, \partial_{i} G_{33}\right)^{t}, \\
G\left(\gamma^{1}, \gamma^{2}, \gamma^{3}\right)^{t}= & G Q_{0}^{-1} \vec{d}_{0}=Q_{0}^{t} \vec{d}_{0}=-\frac{1}{2}\left(\partial_{1} G_{33}, \partial_{2} G_{33}, 0\right)^{t} .
\end{aligned}
$$

In view of (6.2) we then obtain, for all $i, j=1,2$ :

$$
\left(\alpha_{i j}^{1}, \alpha_{i j}^{2}, \alpha_{i j}^{3}\right)=\left(\Gamma_{i j}^{1}, \Gamma_{i j}^{2}, \Gamma_{i j}^{3}\right), \quad\left(\beta_{i}^{1}, \beta_{i}^{2}, \beta_{i}^{3}\right)=\left(\Gamma_{i 3}^{1}, \Gamma_{i 3}^{2}, \Gamma_{i 3}^{3}\right), \quad\left(\gamma^{1}, \gamma^{2}, \gamma^{3}\right)^{t}=\left(\Gamma_{33}^{1}, \Gamma_{33}^{2}, \Gamma_{33}^{3}\right),
$$

so that (6.8) becomes:

$$
\begin{aligned}
& \partial_{i j} y_{0}=\Gamma_{i j}^{1} \partial_{1} y_{0}+\Gamma_{i j}^{2} \partial_{2} y_{0}+\Gamma_{i j}^{3} \vec{b}_{0}, \\
& \partial_{i} \vec{b}_{0}=\Gamma_{i 3}^{1} \partial_{1} y_{0}+\Gamma_{i 3}^{2} \partial_{2} y_{0}+\Gamma_{i 3}^{3} \vec{b}_{0}, \\
& \vec{d}_{0}=\Gamma_{33}^{1} \partial_{1} y_{0}+\Gamma_{33}^{2} \partial_{2} y_{0}+\Gamma_{33}^{3} \vec{b}_{0} .
\end{aligned}
$$

3. We now prove (6.4). Keeping in mind that $Q_{0}^{T} Q_{0}=G$, the scalar products of expressions in $(6.9)$ are:

$$
\begin{aligned}
& \left\langle\partial_{i j} y_{0}, \vec{d}_{0}\right\rangle=\left\langle\Gamma_{i j}^{n} \partial_{n} y_{0}+\Gamma_{i j}^{3} \vec{b}_{0}, \Gamma_{33}^{p} \partial_{p} y_{0}+\Gamma_{33}^{3} \vec{b}_{0}\right\rangle=G_{n p} \Gamma_{i j}^{n} \Gamma_{33}^{p}, \\
& \left\langle\partial_{i} \vec{b}_{0}, \partial_{j} \vec{b}_{0}\right\rangle=\left\langle\Gamma_{i 3}^{n} \partial_{n} y_{0}+\Gamma_{i 3}^{3} \vec{b}_{0}, \Gamma_{j 3}^{p} \partial_{p} y_{0}+\Gamma_{j 3}^{3} \vec{b}_{0}\right\rangle=G_{n p} \Gamma_{i 3}^{n} \Gamma_{j 3}^{p} .
\end{aligned}
$$

exactly as claimed in (6.4). This ends the proof of Theorem 6.2 and also of Theorem 6.1.

\section{TWO EXAMPLES}

In this section we compute the energy $\mathcal{I}_{4}(V, \mathbb{S})$ in the two particular cases of interest:

$$
G\left(x^{\prime}, x_{3}\right)=\operatorname{diag}\left(1,1, \lambda\left(x^{\prime}\right)\right) \quad \text { and } \quad G\left(x^{\prime}, x_{3}\right)=\lambda\left(x^{\prime}\right) \operatorname{Id}_{3} .
$$

Let $\vec{p}$ be as in the definition (3.6). Writing: $\vec{p}=\alpha^{1} \partial_{1} y_{0}+\alpha^{2} \partial_{2} y_{0}+\alpha^{3} \vec{b}_{0}$, we obtain:

$$
G\left(\alpha^{1}, \alpha^{2}, \alpha^{3}\right)^{t}=-\left(\left\langle\partial_{1} V, \vec{b}_{0}\right\rangle,\left\langle\partial_{2} V, \vec{b}_{0}\right\rangle, 0\right)^{t} .
$$

Consequently:

$$
\vec{p}=-G^{1 i}\left\langle\partial_{i} V, \vec{b}_{0}\right\rangle \partial_{1} y_{0}-G^{2 i}\left\langle\partial_{i} V, \vec{b}_{0}\right\rangle \partial_{2} y_{0}-G^{3 i}\left\langle\partial_{i} V, \vec{b}_{0}\right\rangle \vec{b}_{0} .
$$


Lemma 7.1. Let $\lambda: \bar{\Omega} \rightarrow \mathbb{R}$ be smooth and strictly positive. Consider the metric of the form: $G\left(x^{\prime}, x_{3}\right)=\operatorname{diag}\left(1,1, \lambda\left(x^{\prime}\right)\right)$. Then:

(i) $G$ is immersible in $\mathbb{R}^{3}$ if and only if:

$$
M_{\lambda}=\nabla^{2} \lambda-\frac{1}{2 \lambda} \nabla \lambda \otimes \nabla \lambda \equiv 0 \quad \text { in } \Omega,
$$

while the condition $M_{\lambda} \not \equiv 0$ is equivalent to: $c h^{4} \leq \inf E^{h} \leq C h^{4}$.

(ii) The $\Gamma$-limit energy functional $\mathcal{I}_{4}$ in (3.5) becomes:

$$
\begin{aligned}
\forall w \in W^{1,2}\left(\Omega, \mathbb{R}^{2}\right) \quad \forall v \in W^{2,2}(\Omega, \mathbb{R}) & \\
\mathcal{I}_{4}(v, w)= & \frac{1}{2} \int_{\Omega} \mathcal{Q}_{2}\left(\operatorname{sym} \nabla w+\frac{1}{2} \nabla v \otimes \nabla v+\frac{1}{96 \lambda} \nabla \lambda \otimes \nabla \lambda\right) \mathrm{d} x^{\prime} \\
& +\frac{1}{24} \int_{\Omega} \mathcal{Q}_{2}\left(\sqrt{\lambda} \nabla^{2} v\right)+\frac{1}{5760} \int_{\Omega} \mathcal{Q}_{2}\left(M_{\lambda}\right) \mathrm{d} x^{\prime},
\end{aligned}
$$

where $\mathcal{Q}_{2}$ is independent of $x^{\prime}$ and it is defined by $\mathcal{Q}_{2, I d}$ in (3.4).

Proof. Part (i) of the assertion has been shown in [6]. For (ii), note first that:

$$
y_{0}\left(x^{\prime}\right)=x^{\prime} \quad \text { and } \quad Q_{0}=A=\operatorname{diag}(1,1, \sqrt{\lambda}) .
$$

Consequently, directly from (3.4) we see that $\mathcal{Q}_{2, A}=\mathcal{Q}_{2, I d}$, which we denote simply by $\mathcal{Q}_{2}$.

Further, in view of (4.1), every admissible limiting strain $\mathbb{S} \in \mathbb{S}$ has the form $\mathbb{S}=\operatorname{sym} \nabla w$ for some $w \in W^{1,2}\left(\Omega, \mathbb{R}^{2}\right)$. Also, without loss of generality, every admissible limiting displacement $V$ is of the form: $V=(0,0, v)$ for some $v \in W^{2,2}(\Omega, \mathbb{R})$. We now compute, using (1.9), (6.9) and (7.1):

$$
\vec{b}_{0}=\sqrt{\lambda} e_{3}, \quad \overrightarrow{d_{0}}=-\frac{1}{2}\left(\partial_{1} \lambda, \partial_{2} \lambda, 0\right), \quad \vec{p}=-\sqrt{\lambda}\left(\partial_{1} v, \partial_{2} v, 0\right) .
$$

Therefore:

$$
\begin{aligned}
& \left(\nabla \vec{b}_{0}\right)^{t} \nabla \vec{b}_{0}=\frac{1}{4 \lambda} \nabla \lambda \otimes \nabla \lambda, \quad\left(\nabla y_{0}\right)^{t} \nabla \overrightarrow{d_{0}}=-\frac{1}{2} \nabla^{2} \lambda, \\
& \left(\nabla y_{0}\right)^{t} \nabla \vec{p}=-\frac{1}{2 \sqrt{\lambda}} \nabla v \otimes \nabla \lambda-\sqrt{\lambda} \nabla^{2} v, \quad(\nabla V)^{t} \nabla \vec{b}_{0}=\frac{1}{2 \sqrt{\lambda}} \nabla v \otimes \nabla \lambda .
\end{aligned}
$$

This ends the proof of Lemma 7.1 in view of (3.5).

Lemma 7.2. Let $\lambda: \bar{\Omega} \rightarrow \mathbb{R}$ be smooth and strictly positive. Consider the metric $G\left(x^{\prime}, x_{3}\right)=$ $\lambda\left(x^{\prime}\right) \operatorname{Id}_{3}$. Denote $f=\frac{1}{2} \log \lambda$. Then:

(i) Condition (1.7) is equivalent to $\Delta f=0$, which is also equivalent to the immersability of the metric $G_{2 \times 2}$ in $\mathbb{R}^{2}$.

(ii) Under condition (1.7), condition (6.1) can be directly seen as equivalent to $\operatorname{Ric}(G)=0$ and therefore to the immersability of $G$. 
(iii) The $\Gamma$-limit energy functional in (3.5) has the following form:

$$
\begin{aligned}
\mathcal{I}_{4}(V, \mathbb{S})=\frac{1}{2} & \int_{\Omega} e^{-2 f} \mathcal{Q}_{2}\left(\mathbb{S}+\frac{1}{2}(\nabla V)^{t} \nabla V+\frac{1}{24} e^{2 f} \nabla f \otimes \nabla f\right) \mathrm{d} x^{\prime} \\
& +\frac{1}{24} \int_{\Omega} \mathcal{Q}_{2}\left(2 \nabla V_{3} \otimes \nabla f-\nabla^{2} V_{3}-\left\langle\nabla V_{3}, \nabla f\right\rangle \mathrm{Id}_{2}\right) \mathrm{d} x^{\prime} \\
& +\frac{1}{1440} \int_{\Omega} \mathcal{Q}_{2}\left(e^{f} \operatorname{Ric}(G)_{2 \times 2}\right) \mathrm{d} x^{\prime},
\end{aligned}
$$

where $\mathcal{Q}_{2}$ is as in Lemma 7.1, and where $\operatorname{Ric}(G)_{2 \times 2}$ denotes the tangential part of the Ricci curvature tensor of $G$, i.e.:

$$
\operatorname{Ric}(G)_{2 \times 2}=\left[\begin{array}{ll}
R_{11} & R_{12} \\
R_{12} & R_{22}
\end{array}\right]
$$

Proof. The part $(i)$ has been deduced in [6], together with the expression:

$$
\operatorname{Ric}(G)=-\left(\nabla^{2} f-\nabla f \otimes \nabla f\right)^{*}-\left(\Delta f+|\nabla f|^{2}\right) \operatorname{Id}_{3} .
$$

We now consider the case when (1.7) holds. By $(i)$ the metric $G_{2 \times 2}$ is immersible in $\mathbb{R}^{2}$ and in particular $\vec{N}=e_{3}$. Writing $V=\left(V_{1}, V_{2}, V_{3}\right)$, from (1.9), (6.9) and (7.1) we obtain:

$$
\begin{gathered}
\vec{b}_{0}=\sqrt{\lambda} e_{3}, \quad \vec{d}_{0}=-\left(\partial_{1} f \partial_{1} y_{0}+\partial_{2} f \partial_{2} y_{0}\right), \quad \vec{p}=-\frac{1}{\sqrt{\lambda}}\left(\partial_{1} V_{3} \partial_{1} y_{0}+\partial_{2} V_{3} \partial_{2} y_{0}\right) . \\
\left(\nabla \vec{b}_{0}\right)^{t} \nabla \vec{b}_{0}=e^{2 f} \nabla f \otimes \nabla f, \quad(\nabla V)^{t} \nabla \vec{b}_{0}=e^{f} \nabla V_{3} \otimes \nabla f .
\end{gathered}
$$

Further, observe that: $\partial_{i} \vec{d}_{0}=-\left(\partial_{1 i} f \partial_{1} y_{0}+\partial_{2 i} f \partial_{2} y_{0}+\partial_{1} f \partial_{1 i} y_{0}+\partial_{2} f \partial_{2 i} y_{0}\right)$, and so:

$$
\frac{1}{\lambda}\left\langle\partial_{1} y_{0}, \partial_{1} \vec{d}_{0}\right\rangle=-\frac{1}{\lambda}\left(\lambda \partial_{11} f+\frac{1}{2} \partial_{1} \lambda \partial_{1} f+\frac{1}{2} \partial_{2} \lambda \partial_{2} f\right)=-\left(\partial_{11} f+|\nabla f|^{2}\right) .
$$

In the same manner, we arrive at:

$$
\frac{1}{\lambda}\left\langle\partial_{2} y_{0}, \partial_{2} \vec{d}_{0}\right\rangle=-\left(\partial_{22} f+|\nabla f|^{2}\right), \quad \frac{1}{\lambda}\left\langle\partial_{2} y_{0}, \partial_{1} \vec{d}_{0}\right\rangle=-\partial_{12} f, \quad \frac{1}{\lambda}\left\langle\partial_{1} y_{0}, \partial_{2} \vec{d}_{0}\right\rangle=-\partial_{21} f .
$$

Consequently, $\left(\nabla y_{0}\right)^{t} \nabla \vec{d}_{0}$ is already a symmetric matrix, and:

$$
\left(\nabla y_{0}\right)^{t} \nabla \overrightarrow{d_{0}}=-e^{2 f}\left(\nabla^{2} f+|\nabla f|^{2} \operatorname{Id}_{2}\right) .
$$

In particular, under condition $\Delta f=0$, the formula (7.2) yields:

$$
\operatorname{sym}\left(\nabla y_{0}\right)^{t} \nabla \overrightarrow{d_{0}}+\left(\nabla \vec{b}_{0}\right)^{t} \nabla \vec{b}_{0}=e^{2 f} \operatorname{Ric}(G)_{2 \times 2},
$$

which we directly see to be equivalent with $\nabla f=0$ and hence with $\operatorname{Ric}(G)=0$. This establishes $(i i)$.

We now compute the remaining quantities appearing in the expression of $\mathcal{I}_{4}$. Firstly:

$$
\nabla \vec{p}=\frac{1}{2 \lambda^{3 / 2}} \nabla y_{0}\left(\nabla V_{3} \otimes \nabla \lambda\right)-\frac{1}{\sqrt{\lambda}} \nabla y_{0} \nabla^{2} V_{3}-\frac{1}{\sqrt{\lambda}}\left(\partial_{1} V_{3}\left(\partial_{11} y_{0}, \partial_{12} y_{0}\right)+\partial_{2} V_{3}\left(\partial_{12} y_{0}, \partial_{22} y_{0}\right)\right) .
$$

Using the relations between $\left\langle\partial_{i j} y_{0}, \partial_{k} y_{0}\right\rangle$ and $\partial_{l} G$ in (6.5), we obtain:

$$
\left(\nabla y_{0}\right)^{t} \nabla \vec{p}=\frac{1}{2 \lambda^{3 / 2}} G_{2 \times 2} \nabla V_{3} \otimes \nabla \lambda-\frac{1}{\sqrt{\lambda}} G_{2 \times 2} \nabla^{2} V_{3}-\frac{1}{2 \sqrt{\lambda}}\left[\begin{array}{c|c}
\left\langle\nabla V_{3}, \nabla \lambda\right\rangle & \left\langle\nabla V_{3}, \nabla \lambda^{\perp}\right\rangle \\
\hline-\left\langle\nabla V_{3}, \nabla \lambda^{\perp}\right\rangle & \left\langle\nabla V_{3}, \nabla \lambda\right\rangle
\end{array}\right]
$$


and therefore:

$$
\operatorname{sym}\left(\nabla y_{0}\right)^{t} \nabla \vec{p}=\sqrt{\lambda} \operatorname{sym}\left(\nabla V_{3} \otimes \nabla f\right)-\sqrt{\lambda} \nabla^{2} V_{3}-\sqrt{\lambda}\left\langle\nabla V_{3}, \nabla \lambda\right\rangle \operatorname{Id}_{2} .
$$

In a similar manner, it follows that:

$$
\operatorname{sym}\left(\nabla y_{0}\right)^{t} \nabla \overrightarrow{d_{0}}=-\lambda\left(\nabla^{2} f+|\nabla f|^{2} \operatorname{Id}_{2}\right) .
$$

Since $\mathcal{Q}_{2, A}\left(x^{\prime}\right)=\lambda^{-1} \mathcal{Q}_{2}$, the formula in (3.5) becomes:

$$
\begin{aligned}
\mathcal{I}_{4}(V, \mathbb{S})= & \frac{1}{2} \int_{\Omega} e^{-2 f} \mathcal{Q}_{2}\left(\mathbb{S}+\frac{1}{2}(\nabla V)^{t} \nabla V+\frac{1}{24} e^{2 f} \nabla f \otimes \nabla f\right) \mathrm{d} x^{\prime} \\
& +\frac{1}{24} \int_{\Omega} e^{-2 f} \mathcal{Q}_{2}\left(2 e^{f} \nabla V_{3} \otimes \nabla f-e^{f} \nabla^{2} V_{3}-e^{f}\left\langle\nabla V_{3}, \nabla f\right\rangle \mathrm{Id}_{2}\right) \mathrm{d} x^{\prime} \\
& +\frac{1}{1440} \int_{\Omega} e^{-2 f} \mathcal{Q}_{2}\left(e^{2 f} \operatorname{Ric}(G)_{2 \times 2}\right) \mathrm{d} x^{\prime},
\end{aligned}
$$

which implies the result.

\section{Appendix: A proof of Corollary 2.3}

1. For every $x^{\prime} \in \Omega$ denote $D_{x^{\prime}, \delta}=B\left(x^{\prime}, \delta\right) \cap \Omega$ and $B_{x^{\prime}, \delta, h}=D_{x^{\prime}, \delta} \times(-h / 2, h / 2)$. For short, we write $B_{x^{\prime}, 2 h}=B_{x^{\prime}, 2 h, h}$ and $B_{x^{\prime}, h}=B_{x^{\prime}, h, h}$. Apply Lemma 2.2 to the set $\mathcal{V}^{h}=B_{x^{\prime}, 2 h}$ to get a rotation $R_{x^{\prime}, 2 h} \in S O(3)$ such that, with a universal constant $C$ :

$$
\begin{aligned}
\frac{1}{h} \int_{B_{x^{\prime}, 2 h}} \mid \nabla u^{h}(z)-R_{x^{\prime}, 2 h}\left(Q_{0}\left(z^{\prime}\right)+\right. & \left.z_{3} B_{0}\left(z^{\prime}\right)\right)\left.\right|^{2} \mathrm{~d} z \\
& \leq C\left(E^{h}\left(u^{h}, B_{x^{\prime}, 2 h}\right)+h^{3}\left|B_{x^{\prime}, 2 h}\right|\right) .
\end{aligned}
$$

Consider a family of mollifiers $\eta_{x^{\prime}} \in C^{\infty}(\Omega, \mathbb{R})$, parametrized by $x^{\prime} \in \Omega$ :

$$
\int_{\Omega} \eta_{x^{\prime}}=\frac{1}{h}, \quad\left\|\eta_{x^{\prime}}\right\|_{L^{\infty}(\Omega)} \leq \frac{C}{h^{3}}, \quad\left\|\nabla_{x^{\prime}} \eta_{x^{\prime}}\right\|_{L^{\infty}(\Omega)} \leq \frac{C}{h^{4}} \quad \text { and }\left(\operatorname{supp} \eta_{x^{\prime}}\right) \cap \Omega \subset D_{x^{\prime}, h} .
$$

Define $\tilde{R}^{h} \in W^{1,2}\left(\Omega, \mathbb{R}^{3 \times 3}\right)$ as:

$$
\tilde{R}^{h}\left(x^{\prime}\right)=\int_{\Omega^{h}} \eta_{x^{\prime}}\left(z^{\prime}\right) \nabla u^{h}(z)\left(Q_{0}\left(z^{\prime}\right)+z_{3} B_{0}\left(z^{\prime}\right)\right)^{-1} \mathrm{~d} z .
$$

We then have:

$$
\begin{aligned}
& \frac{1}{h} \int_{B_{x^{\prime}, h}}\left|\nabla u^{h}(z)-\tilde{R}^{h}\left(z^{\prime}\right)\left(Q_{0}\left(z^{\prime}\right)+z_{3} B_{0}\left(z^{\prime}\right)\right)\right|^{2} \mathrm{~d} z \\
& \leq \frac{C}{h} \int_{B_{x^{\prime}, 2 h}}\left|\nabla u^{h}(z)-R_{x^{\prime}, 2 h}\left(Q_{0}\left(z^{\prime}\right)+z_{3} B_{0}\left(z^{\prime}\right)\right)\right|^{2} \mathrm{~d} z \\
&+\frac{C}{h} \int_{B_{x^{\prime}, h}}\left|\tilde{R}^{h}\left(z^{\prime}\right)-R_{x^{\prime}, 2 h}\right|^{2}\left|Q_{0}\left(z^{\prime}\right)+z_{3} B_{0}\left(z^{\prime}\right)\right|^{2} \mathrm{~d} z \\
& \leq C\left(E^{h}\left(u^{h}, B_{x^{\prime}, 2 h}\right)+h^{3}\left|B_{x^{\prime}, 2 h}\right|\right)+\frac{C}{h} \int_{B_{x^{\prime}, h}}\left|\tilde{R}^{h}\left(z^{\prime}\right)-R_{x^{\prime}, 2 h}\right|^{2} \mathrm{~d} z,
\end{aligned}
$$


where we have used (8.1) and $\left\|Q_{0}\left(z^{\prime}\right)+z_{3} B_{0}\left(z^{\prime}\right)\right\|_{L^{\infty}} \leq C$. Now, for every $z^{\prime} \in B_{x^{\prime}, h}$ we have:

$$
\begin{aligned}
& \left|\tilde{R}^{h}\left(z^{\prime}\right)-R_{x^{\prime}, 2 h}\right|^{2}=\left|\int_{\Omega^{h}} \eta_{z^{\prime}}\left(y^{\prime}\right) \nabla u^{h}(y)\left(Q_{0}\left(y^{\prime}\right)+y_{3} B_{0}\left(y^{\prime}\right)\right)^{-1} \mathrm{~d} y-R_{x^{\prime}, 2 h}\right|^{2} \\
& \quad=\left|\int_{\Omega^{h}} \eta_{z^{\prime}}\left(y^{\prime}\right)\left(\nabla u^{h}(y)-R_{x^{\prime}, 2 h}\left(Q_{0}\left(y^{\prime}\right)+y_{3} B_{0}\left(y^{\prime}\right)\right)\right)\left(Q_{0}\left(z^{\prime}\right)+y_{3} B_{0}\left(z^{\prime}\right)\right)^{-1} \mathrm{~d} y\right|^{2} \\
& \leq C\left(\int_{B_{z^{\prime}, h}} \eta_{z^{\prime}}\left(y^{\prime}\right)^{2} \mathrm{~d} y\right)\left(\int_{B_{z^{\prime}, h}}\left|\nabla u^{h}(y)-R_{x^{\prime}, 2 h}\left(Q_{0}\left(y^{\prime}\right)+y_{3} B_{0}\left(y^{\prime}\right)\right)\right|^{2} \mathrm{~d} y\right) \\
& \leq \frac{C}{h^{2}} \int_{B_{x^{\prime}, 2 h}}\left|\nabla u^{h}(y)-R_{x^{\prime}, 2 h}\left(Q_{0}\left(y^{\prime}\right)+y_{3} B_{0}\left(y^{\prime}\right)\right)\right|^{2} \mathrm{~d} y \\
& \leq \frac{C}{h^{2}}\left(E^{h}\left(u^{h}, B_{x^{\prime}, 2 h}\right)+h^{3}\left|B_{x^{\prime}, 2 h}\right|\right) .
\end{aligned}
$$

In a similar way, in view of $\int_{\Omega^{h}} \nabla_{z^{\prime}} \eta_{z^{\prime}}\left(y^{\prime}\right) \mathrm{d} y=0$, it follows that:

$$
\begin{aligned}
\left|\nabla \tilde{R}^{h}\left(z^{\prime}\right)\right|^{2} & =\left(\int_{\Omega^{h}} \nabla_{z^{\prime}} \eta_{z^{\prime}}\left(y^{\prime}\right) \nabla u^{h}(y)\left(Q_{0}\left(y^{\prime}\right)+y_{3} B_{0}\left(y^{\prime}\right)\right)^{-1} \mathrm{~d} y\right)^{2} \\
& =\left(\int_{B_{x^{\prime}, 2 h}} \nabla_{z^{\prime}} \eta_{z^{\prime}}\left(y^{\prime}\right)\left(\nabla u^{h}(y)\left(Q_{0}\left(y^{\prime}\right)+y_{3} B_{0}\left(y^{\prime}\right)\right)^{-1}-R_{x^{\prime}, 2 h}\right) \mathrm{d} y\right)^{2} \\
& \leq C \int_{\Omega^{h}}\left|\nabla_{z^{\prime}} \eta_{z^{\prime}}\left(y^{\prime}\right)\right|^{2} \mathrm{~d} y \int_{B_{x^{\prime}, 2 h}}\left|\nabla u^{h}(y)-R_{x^{\prime}, 2 h}\left(Q_{0}\left(y^{\prime}\right)+y_{3} B_{0}\left(y^{\prime}\right)\right)\right|^{2} \mathrm{~d} y \\
& \leq \frac{C}{h^{4}}\left(E^{h}\left(u^{h}, B_{x^{\prime}, 2 h}\right)+h^{3}\left|B_{x^{\prime}, 2 h}\right|\right) .
\end{aligned}
$$

From (8.4) we obtain:

$$
\begin{aligned}
\int_{B_{x^{\prime}, h}}\left|\tilde{R}^{h}\left(z^{\prime}\right)-R_{x^{\prime}, 2 h}\right|^{2} \mathrm{~d} z & \leq \frac{C}{h^{2}} \int_{B_{x^{\prime}, h}}\left(E^{h}\left(u^{h}, B_{x^{\prime}, 2 h}\right)+h^{4}\left|B_{x^{\prime}, 2 h}\right|\right) \mathrm{d} z \\
& \leq C h\left(E^{h}\left(u^{h}, B_{x^{\prime}, 2 h}\right)+h^{3}\left|B_{x^{\prime}, 2 h}\right|\right)
\end{aligned}
$$

and therefore by (8.3) we further see that:

$$
\begin{aligned}
\frac{1}{h} \int_{B_{x^{\prime}, h}} \mid \nabla u^{h}(z)-\tilde{R}^{h}\left(z^{\prime}\right)\left(Q_{0}\left(z^{\prime}\right)\right. & \left.+z_{3} B_{0}\left(z^{\prime}\right)\right)\left.\right|^{2} \mathrm{~d} z \\
& \leq C\left(E^{h}\left(u^{h}, B_{x^{\prime}, 2 h}\right)+h^{3}\left|B_{x^{\prime}, 2 h}\right|\right) .
\end{aligned}
$$

2. Covering $\Omega^{h}$ by a finite family of sets $\left\{B_{x^{\prime}, h}\right\}$, such that the intersection number of the doubled covering $\left\{B_{x^{\prime}, 2 h}\right\}$ is independent of $h$, applying (8.5) and summing over the covering, it follows that:

$$
\frac{1}{h} \int_{\Omega^{h}}\left|\nabla u^{h}(z)-\tilde{R}^{h}\left(z^{\prime}\right)\left(Q_{0}\left(z^{\prime}\right)+z_{3} B_{0}\left(z^{\prime}\right)\right)\right|^{2} \mathrm{~d} z \leq C\left(E^{h}\left(u^{h}\right)+h^{4}\right) .
$$


In a similar fashion we obtain:

$$
\begin{aligned}
\int_{D_{x^{\prime}, h}}\left|\nabla \tilde{R}^{h}\left(z^{\prime}\right)\right|^{2} \mathrm{~d} z & \leq \frac{C}{h^{4}} \int_{D_{x^{\prime}, h}}\left(E^{h}\left(u^{h}, B_{x^{\prime}, 2 h}\right)+h^{3}\left|B_{x^{\prime}, 2 h}\right|\right) \mathrm{d} z \\
& \leq \frac{C}{h^{2}}\left(E^{h}\left(u^{h}, B_{x^{\prime}, 2 h}\right)+h^{3}\left|B_{x^{\prime}, 2 h}\right|\right),
\end{aligned}
$$

and by the same covering argument:

$$
\int_{\Omega^{h}}\left|\nabla \tilde{R}^{h}\left(z^{\prime}\right)\right|^{2} \mathrm{~d} z \leq \frac{C}{h^{2}}\left(E^{h}\left(u^{h}\right)+h^{4}\right) .
$$

3. Note that, in the above two estimates, we can replace $\tilde{R}^{h}$ by $R^{h}=\mathbb{P}_{\mathrm{SO}(3)} \tilde{R}^{h} \in$ $W^{1,2}(\Omega, S O(3))$. Firstly, the projection in question is well defined in view of $(8.4)$, since:

$$
\operatorname{dist}^{2}\left(\tilde{R}^{h}, S O(3)\right) \leq\left|\tilde{R}^{h}-R_{x^{\prime}, 2 h}\right| \leq \frac{C}{h^{2}}\left(E^{h}\left(u^{h}\right)+h^{4}\right),
$$

which is small because of the hypothesis $\alpha<2$. Moreover:

$$
\begin{aligned}
\frac{1}{h} \int_{B_{x^{\prime}, h}} \mid \nabla u^{h}(z)-R^{h}\left(z^{\prime}\right) & \left.\left(Q_{0}\left(z^{\prime}\right)+z_{3} B_{0}\left(z^{\prime}\right)\right)\right|^{2} \mathrm{~d} z \\
\leq & \frac{C}{h} \int_{B_{x^{\prime}, h}}\left|\nabla u^{h}(z)-\tilde{R}^{h}\left(z^{\prime}\right)\left(Q_{0}\left(z^{\prime}\right)+z_{3} B_{0}\left(z^{\prime}\right)\right)\right|^{2} \mathrm{~d} z \\
& +\frac{C}{h} \int_{B_{x^{\prime}, h}}\left|\tilde{R}^{h}\left(z^{\prime}\right)-R^{h}\left(z^{\prime}\right)\right|^{2}\left|Q_{0}\left(z^{\prime}\right)+z_{3} B_{0}\left(z^{\prime}\right)\right|^{2} \mathrm{~d} z \\
\leq & C\left(E^{h}\left(u^{h}, B_{x^{\prime}, 2 h}\right)+h^{3}\left|B_{x^{\prime}, 2 h}\right|\right)
\end{aligned}
$$

because of (8.5) and (8.4). Finally, the previous covering argument clearly implies (2.9), and $\int_{\Omega}\left|\nabla R^{h}\right|^{2} \mathrm{~d} z \leq C \int_{\Omega}\left|\nabla \tilde{R}^{h}\right|^{2} \mathrm{~d} z$ yields (2.10).

\section{REFERENCES}

[1] E. Acerbi, G. Buttazzo and D. Percivale, A variational definition for the strain energy of an elastic string, J. Elasticity 25 (1991), 137-148.

[2] B. Audoly and A. Boudaoud, Self-similar structures near boundaries in strained systems, Phys. Rev. Lett. 91 (2004), 086105-086108.

[3] B. Barker, M. Lewicka and K. Zumbrun, Existence and stability of viscoelastic shock profiles, Arch. Rational Mech. Anal. 200 (2011), 491-532.

[4] P. Bella and R.V. Kohn, Metric-induced wrinkling of a thin elastic sheet, J. Nonlinear Sci. 24 (2014), 11471176.

[5] M. Ben Amar, M.M. Müller, M. Trejo, Petal shapes of sympetalous flowers: the interplay between growth, geometry and elasticity, New J. Physics 14 (2012), 085014.

[6] K. Bhattacharya, M. Lewicka and M. Schaffner, Plates with incompatible prestrain, to appear in Arch. Rational. Mech. Anal.

[7] D. Caillerie, E. Sanchez-Palencia, A new kind of singular stiff problems and application to thin elastic shells, Math. Models Methods Appl. Sci. 5 (1995), 47- 66.

[8] D. Caillerie, E. Sanchez-Palencia, Elastic thin shells: asymptotic theory in the anisotropic and heterogeneous cases, Math. Models Methods Appl. Sci. 5 (1995), 473- 496. 
[9] P.G. Ciarlet, A justification of the von Kármán equations, Arch. Rational Mech. Anal. 73 (1980), 349-389.

[10] P.G. Ciarlet, Mathematical Elasticity, Vol. II: Theory of Plates, North-Holland, Amsterdam (1997).

[11] P.G. Ciarlet, Mathematical Elasticity, Vol. III: Theory of Shells, North-Holland, Amsterdam (2000).

[12] P.G. Ciarlet, P. Destuynder, A justification of the two-dimensional linear plate model, J. Mécanique 18 (1979), 315-344.

[13] P.G. Ciarlet, P. Destuynder, A justification of a nonlinear model in plate theory, Methods Appl. Mech. Engrg. 1718 (1979), 227-258.

[14] P.G. Ciarlet, V. Lods, Asymptotic analysis of linearly elastic shells. I. Justification of membrane shells equations, Arch. Rational Mech. Anal. 136 (1996), 119-161.

[15] P.G. Ciarlet, V. Lods, Asymptotic analysis of linearly elastic shells : Generalized membrane shells, J. Elasticity 43 (1996), 147-188.

[16] P.G. Ciarlet, V. Lods, B. Miara, Asymptotic analysis of linearly elastic shells. II. Justification of flexural shells, Arch. Rational Mech. Anal. 136 (1996), 163-190.

[17] S. Conti and G. Dolzmann, Derivation of elastic theories for thin sheets and the constraint of incompressibility, Analysis, modeling and simulation of multiscale problems, 225-247, Springer, Berlin, (2006).

[18] S. Conti and G. Dolzmann, Derivation of a plate theory for incompressible materials, C. R. Math. Acad. Sci. Paris 344 (2007), 541-544.

[19] S. Conti and F. Maggi, Confining thin sheets and folding paper, Arch. Ration. Mech. Anal. 187 (2008), 1-48.

[20] J. Dervaux, M. Ben Amar, Morphogenesis of growing soft tissues, Phys. Rev. Lett. 101 (2008), 068101.

[21] J. Dervaux, P. Ciarletta, and M. Ben Amar, Morphogenesis of thin hyperelastic plates: a constitutive theory of biological growth in the Foppl-von Karman limit, J. Mech. Phys. Solids 57 (2009), 458-471.

[22] P. Destuynder, Sur une justification des modèles de plaques et de coques par les méthodes asymptotiques, Doctorat d'état, Paris (1980).

[23] P. Destuynder, Comparaison entre les modèles tri-dimensionnels et bi-dimensionnels de plaques en élasticité, RAIRO Analyse Numérique 15 (1981), 331-369.

[24] P. Destuynder, A classification of thin shell theories, Acta Appl. Math. 4 (1985), 15-63.

[25] E. Efrati, E. Sharon, and R. Kupferman, Elastic theory of unconstrained non-Euclidean plates J. Mech. Phys. Solids, 57 (2009), 762-775.

[26] D.D. Fox, A. Raoult and J.C. Simo, A justification of nonlinear properly invariant plate theories, Arch. Rational Mech. Anal. 124 (1993), 157-199.

[27] G. Friesecke, R. James, M.G. Mora and S. Müller, Derivation of nonlinear bending theory for shells from three-dimensional nonlinear elasticity by Gamma-convergence, C. R. Math. Acad. Sci. Paris, 336 (2003), 697-702.

[28] G. Friesecke, R. James and S. Müller, A theorem on geometric rigidity and the derivation of nonlinear plate theory from three dimensional elasticity, Comm. Pure Appl. Math. 55 (2002), 1461-1506.

[29] G. Friesecke, R. James and S. Müller, A hierarchy of plate models derived from nonlinear elasticity by Gamma-convergence, Arch. Ration. Mech. Anal. 180 (2006), 183-236.

[30] M. Gurtin, E. Fried and L. Anand, The mechanics and thermodynamics of continua, Cambridge University Press, Cambridge (2010).

[31] Q. Han and J.X. Hong, Isometric embedding of Riemannian manifolds in Euclidean spaces, Mathematical Surveys and Monographs 130, American Mathematical Society, Providence, RI (2006).

[32] P. Hornung, M. Lewicka and R. Pakzad, Infinitesimal isometries on developable surfaces and asymptotic theories for thin developable shells, J. Elasticity 111 (2013)

[33] Y. Klein, E. Efrati and E. Sharon, Shaping of elastic sheets by prescription of non-Euclidean metrics, Science 315 (2007), 1116-1120.

[34] H. Le Dret and A. Raoult, The nonlinear membrane model as a variational limit of nonlinear threedimensional elasticity, J. Math. Pures Appl. 73 (1995), 549-578. 
[35] H. Le Dret and A. Raoult, The membrane shell model in nonlinear elasticity: a variational asymptotic derivation, J. Nonlinear Sci. 6 (1996), 59-84.

[36] M. Lewicka, A note on the convergence of low energy critical points of nonlinear elasticity functionals, for thin shells of arbitrary geometry, ESAIM: Control, Optimisation and Calculus of Variations 17 (2011), 493-505.

[37] M. Lewicka and H. Li, Convergence of equilibria for incompressible elastic plates in the von Karman regime, Comm. Pure Appl. Anal. 14, Issue 1 (January 2015)

[38] M. Lewicka, L. Mahadevan and R. Pakzad, The Foppl-von Karman equations for plates with incompatible strains, Proc. Roy. Soc. A 467 (2011), 402-426.

[39] M. Lewicka, L. Mahadevan and R. Pakzad, Models for elastic shells with incompatible strains, Proc. Roy. Soc. A 47 (2014), 2165 20130604; 1471-2946.

[40] M. Lewicka, L. Mahadevan and R. Pakzad, The Monge-Ampere constrained elastic theories of shallow shells, to appear.

[41] M. Lewicka, M.G. Mora and R. Pakzad, Shell theories arising as low energy Gamma-limit of 3d nonlinear elasticity, Ann. Scuola Norm. Sup. Pisa Cl. Sci. (5) Vol. IX (2010), 1-43.

[42] M. Lewicka, M.G. Mora and R. Pakzad, The matching property of infinitesimal isometries on elliptic surfaces and elasticity of thin shells, Arch. Rational Mech. Anal. (3), 200 (2011), 1023-1050.

[43] M. Lewicka, P. Ochoa and R. Pakzad, Variational models for prestrained plates with Monge-Ampere constraint, to appear.

[44] M. Lewicka and R. Pakzad, Scaling laws for non-Euclidean plates and the $W^{2,2}$ isometric immersions of Riemannian metrics, ESAIM: Control, Optimisation and Calculus of Variations, 17 (2011), 11581173.

[45] M. Lewicka and M. Pakzad, The infinite hierarchy of elastic shell models; some recent results and a conjecture, Infinite Dimensional Dynamical Systems, Fields Institute Communications 64 (2013) 407-420.

[46] H. Li, The Kirchhoff theory for elastic pre-strained shells, Nonlinear Analysis: Theory, Methods \& Applications, 78 (2013) 1-16.

[47] H. Li and M. Chermisi, The von Karman theory for incompressible elastic shells, Calculus of Variations and Partial Differential Equations 48 1-2 (2013) 185-209.

[48] H. Liang and L. Mahadevan, The shape of a long leaf, Proc. Nat. Acad. Sci. (2009).

[49] F. C. Liu, A Lusin property of Sobolev functions, Indiana U. Math. J. 26 (1977) 645-651.

[50] B. Miara, E. Sanchez-Palencia, Asymptotic analysis of linearly elastic shells, Asymptotic Analysis 12 (1996), 41-54.

[51] C.D. Modes, K. Bhattacharya and M. Warner, Gaussian curvature from flat elastica sheets, Proc. Roy. Soc. A 467 (2011), 1121-1140.

[52] R. Monneau, Justification of nonlinear Kirchhoff-Love theory of plates as the application of a new singular inverse method, Arch. Rational Mech. Anal. 169 (2003), 1-34.

[53] M.G. Mora and L. Scardia, Convergence of equilibria of thin elastic plates under physical growth conditions for the energy density, J. Differential Equations 252 (2012), 35-55.

[54] S. Müller and M. R. Pakzad, Convergence of equilibria of thin elastic plates - the von Kármán case, Comm. Partial Differential Equations 33 (2008), 1018 - 1032.

[55] A. Raoult, Construction d'un modèle d'évolution de plaques avec terme d'inertie de rotation, Ann. Mat. Pura Appl. CXXXIX (1985), 361-400.

[56] A. Raoult, Analyse mathématique de quelques modèles de plaques et de poutres élastiques ou élastoplastiques, Doctorat d'état, Paris (1988).

[57] E.K. Rodriguez, A. Hoger and A. McCulloch, Stress-dependent finite growth in finite soft elatic tissues J. Biomechanics 27 (1994), 455-467.

[58] E. Sanchez-Palencia, Passage à la limite de l'élasticité tridimensionnelle à la théorie asymptotique des coques minces, C. R. Acad. Sci. Paris Sér. II 311 (1990), 909-916.

[59] E. Sanchez-Palencia, Statique et dynamique des coques minces. I. Cas de flexion pure non inhibée, C. R. Acad. Sci. Paris Sér. I 309 (1989), 411-417. 
[60] E. Sanchez-Palencia, Statique et dynamique des coques minces. II. Cas de flexion pure inhibée. Approximation membranaire, C. R. Acad. Sci. Paris Sér. I Math. 309 (1989), 531-537.

[61] B. Schmidt, Plate theory for stressed heterogeneous multilayers of finite bending energy, J. Math. Pures Appl. 88 (2007), 107-122.

[62] E. Sharon, B. Roman, H.L. Swinney, Geometrically driven wrinkling observed in free plastic sheets and leaves, Phys. Rev. E 75 (2007), 046211-046217.

[63] K. Trabelsi, Modeling of a membrane for nonlinearly elastic incompressible materials via Gammaconvergence, Anal. Appl. (Singap.) 4 (2006), 31-60.

Marta Lewicka, University of Pittsburgh, Department of Mathematics, 301 Thackeray Hall, PitTsburgh, PA 15260, USA

E-mail address: lewicka@pitt.edu

Annie Raoult, Laboratoire MaP5, Université Paris Descartes \& CNRS, Sorbonne Paris Cité, FRANCE

E-mail address: annie.raoult@parisdescartes.fr

Diego Ricciotti, University of Pittsburgh, Department of Mathematics, 301 Thackeray Hall, PitTsburgh, PA 15260, USA

E-mail address: DIR17@pitt.edu 